\title{
Nestin Selectively Facilitates the Phosphorylation of the Lissencephaly-Linked Protein Doublecortin (DCX) by cdk5/p35 to Regulate Growth Cone Morphology and Sema3a Sensitivity in Developing Neurons
}

\author{
${ }^{\circledR}$ Christopher J. Bott, ${ }^{1}$ Lloyd P. McMahon, ${ }^{1 *}{ }^{\oplus}$ Jason M. Keil, ${ }^{2,3 *}{ }^{\circledR}$ Chan Choo Yap, ${ }^{1}{ }^{\circledR}$ Kenneth Y. Kwan, ${ }^{2,3}$ and \\ ${ }^{-}$Bettina Winckler ${ }^{1}$ \\ ${ }^{1}$ Department of Cell Biology, University of Virginia, Charlottesville, Virginia 22908, ${ }^{2}$ Michigan Neuroscience Institute (MNI), and ${ }^{3}$ Department of \\ Human Genetics, University of Michigan, Ann Arbor, Michigan 48109
}

\begin{abstract}
Nestin, an intermediate filament protein widely used as a marker of neural progenitors, was recently found to be expressed transiently in developing cortical neurons in culture and in developing mouse cortex. In young cortical cultures, nestin regulates axonal growth cone morphology. In addition, nestin, which is known to bind the neuronal cdk5/p35 kinase, affects responses to axon guidance cues upstream of cdk5, specifically, to Sema3a. Changes in growth cone morphology require rearrangements of cytoskeletal networks, and changes in microtubules and actin filaments are well studied. In contrast, the roles of intermediate filament proteins in this process are poorly understood, even in cultured neurons. Here, we investigate the molecular mechanism by which nestin affects growth cone morphology and Sema3a sensitivity. We find that nestin selectively facilitates the phosphorylation of the lissencephaly-linked protein doublecortin (DCX) by cdk5/p35, but the phosphorylation of other cdk5 substrates is not affected by nestin. We uncover that this substrate selectivity is based on the ability of nestin to interact with DCX, but not with other cdk5 substrates. Nestin thus creates a selective scaffold for DCX with activated cdk5/p35. Last, we use cortical cultures derived from $D c x$ KO mice to show that the effects of nestin on growth cone morphology and on Sema3a sensitivity are DCX-dependent, thus suggesting a functional role for the DCX-nestin complex in neurons. We propose that nestin changes growth cone behavior by regulating the intracellular kinase signaling environment in developing neurons. The sex of animal subjects is unknown.
\end{abstract}

Key words: axon guidance; CDK5; DCX; growth cone; intermediate filaments; nestin

\section{Significance Statement}

Nestin, an intermediate filament protein highly expressed in neural progenitors, was recently identified in developing neurons where it regulates growth cone morphology and responsiveness to the guidance cue Sema3a. Changes in growth cone morphology require rearrangements of cytoskeletal networks, but the roles of intermediate filaments in this process are poorly understood. We now report that nestin selectively facilitates phosphorylation of the lissencephaly-linked doublecortin (DCX) by cdk5/ $\mathrm{p} 35$, but the phosphorylation of other cdk5 substrates is not affected. This substrate selectivity is based on preferential scaffolding of DCX, cdk5, and p35 by nestin. Additionally, we demonstrate a functional role for the DCX-nestin complex in neurons. We propose that nestin changes growth cone behavior by regulating intracellular kinase signaling in developing neurons.

Received Oct. 17, 2019; revised Feb. 24, 2020; accepted Mar. 24, 2020.

Author contributions: C.J.B., J.M.K., C.C.Y., K.Y.K., and B.W. designed research; C.J.B. and J.M.K. performed research; C.J.B., J.M.K., and K.Y.K. contributed unpublished reagents/analytic tools; C.J.B. and L.P.M. analyzed data; C.J.B. wrote the first draft of the paper; C.J.B., L.P.M., J.M.K., C.C.Y., K.Y.K., and B.W. edited the paper; C.J.B. wrote the paper.

This work was supported by NIH grant R01NS081674 (to B.W.) and R01 NS097525 (to K.Y.K). C.J.B. was supported by an institutional NIH training grant (T32 GM008136) and J.M.K. by F30 MH112328 and T32 GM007544. We thank the Deppman laboratory for TUJ1 antibody; Laura Digilio for assistance with editing and Airyscan imaging expertise; Ashley Mason for assistance with editing; the Kipnis laboratory for use of Amaxa 4d electroporator; Lawrence Brass (University of Pennsylvania) for Myc-SPN expression construct; Bruce Lahn's laboratory (University of Chicago) for generously providing mouse nestin CDNA; Dr. Martin Balastik (Cech Academy of Sciences) for generously providing the CRMP2 reagents; Clay Hazlett (Nikon) for assistance with the Nikon TiE enhanced confocal imaging; and Dr. Judy Liu (Brown University) for the DCX KO mice.

\section{Introduction}

Correct wiring of the brain requires the targeting of growing axons to their future target fields. The outgrowth of axons is driven by the growth cone (GC), which is an actin- and 
microtubule (MT)-rich motile structure at the tip of growing axons (Menon and Gupton, 2016; Miller and Suter, 2018). GCs also respond directionally to a large number of extracellular guidance cues (Yamamoto et al., 2002; Petrovic and Schmucker, 2015). Guidance cues elicit local signaling responses, which result in rearrangement of the GC cytoskeleton to give directional growth (Dent et al., 2011; Vitriol and Zheng, 2012), especially via guidance cue-activated kinases and their downstream substrates (Dent et al., 2011). Phosphorylation of cytoskeleton-associated proteins downstream of guidance cues is thus an important mechanism by which incoming guidance cue information is converted into morphologic rearrangements of the GC.

Most work on the cytoskeleton of axonal GCs has focused on actin filaments and MTs (Yamada et al., 1970; Dent and Gertler, 2003; Geraldo and Gordon-Weeks, 2009; Kapitein and Hoogenraad, 2015; Tamariz and Varela-Echavarría, 2015). Distinct roles for the third main cytoskeletal filament system, the intermediate filaments (IFs), during early axon formation and GC guidance are not extensively studied (Bott and Winckler, 2020), despite early promising studies in Xenopus neurons (Szaro et al., 1991; Lin and Szaro, 1995, 1996; Walker et al., 2001). IFs are sparsely expressed in growing axons and are composed of vimentin and $\alpha$-internexin (INA) before expression of the other neurofilaments NF-L, NF-M, and NF-H (Shaw et al., 1985; Pennypacker et al., 1991; Benson et al., 1996; L. Wang et al., 2000; Chang and Goldman, 2004; Uchida and Brown, 2004; Brown, 2013). Once axons reach their targets, NF-M and NF-H are greatly upregulated (Nixon and Shea, 1992; Yabe et al., 2003; Laser-Azogui et al., 2015).

The atypical IF protein nestin is highly expressed in neuronal progenitor cells. We recently found lower levels of nestin protein in postmitotic cortical neurons, especially in the distal axon. Depletion of axonal nestin leads to increased GC size and blunts sensitivity to the cdk5-dependent axonal guidance cue Sema3a (Bott et al., 2019). Our previous study thus was the first to discover a role for nestin in neurons (Bott et al., 2019), but the molecular pathways by which nestin affects GCs remains unknown. Nestin binds the cdk5/p35 heterodimer complex (Sahlgren et al., 2003, 2006; Yang et al., 2011), but the cdk5 substrates affected by nestin are not known. The best studied example of nestin-mediated regulation of $c \mathrm{dk} 5$ is at the neuromuscular junction (Yang et al., 2011), but even there the physiological substrates of cdk5 affected by nestin are still unknown. In this work, we discover a neuronal nestin-regulated cdk5 substrate (i.e., doublecortin [DCX]) and show that nestin's effect on GC morphology is DCX-dependent.

DCX is a cytoskeleton-associated protein expressed in developing neurons (Gleeson, et al., 1999; Sapir et al., 2000; Tanaka et al., 2004; Tsukada et al., 2005; Ge et al., 2006; Yap et al., 2012, 2018; Moslehi et al., 2017). Mutations in human DCX cause lissencephaly, a disease characterized by defects in neuronal migration and axon outgrowth that results in profound cortical malformations (Bahi-Buisson et al., 2013). Patients exhibit intellectual disability and intractable epilepsy and often die in childhood. In vitro, DCX promotes MT assembly, and DCX-bound MTs are less dynamic (Moores et al., 2004). Multiple kinases regulate DCX phosphorylation (Schaar et al., 2004; Tanaka et al., 2004), including cdk5 (Xie et al., 2006; Nishimura et al., 2014). Phosphorylation of DCX by cdk 5 decreases the affinity of DCX for MTs and thus leads to decreased MT stability and increased MT dynamics (Tanaka et al., 2004; Bielas et al., 2007).

In this work, we identify DCX as a novel nestin-associated protein and demonstrate that cdk5 potently phosphorylates nestin-scaffolded DCX. In addition, we show that nestin's effects on GC morphology and Sema3a sensitivity are DCX-dependent, thus demonstrating a functional role for the DCX-nestin complex in neurons.

\section{Materials and Methods}

\section{3 cell culture and transfection}

HEK293 cells were maintained in DMEM $+10 \%$ FBS, and all transfections were conducted using Lipofectamine 2000 (Invitrogen) according to the manufacturer's protocol. Phosphorylation assays and immunoprecipitations were performed on low-passage HEK293s in $6 \mathrm{~cm}$ plates. Cells in low serum (4\%) were transfected for $24-26 \mathrm{~h}$, and serum starved for $10-14 \mathrm{~h}$ before lysis, to reduce background activity of mitotic kinases. Total transfection time was $34-40 \mathrm{~h}$.

\section{Cos-7 cell culture and transfection}

Cos-7 cells were maintained in DMEM $+10 \%$ FBS, and all transfections were conducted using Lipofectamine 2000 (Invitrogen) according to the manufacturer's protocol. Cells were plated on ethanol soaked, washed, round glass Cell Gro Fisher brand coverslip in each 12-well plate well, at a density of 40,000 per well/coverslip; $2 \mu \mathrm{g}$ total of plasmid was used per transfection, and was conducted with equivalent amounts of GFP or GFP tagged construct, and empty vector (EV) myc or myc-tagged construct. The EV myc control is for a c-terminal myc tagged protein, thus has no start codon and is not expected to make any myc protein. Cells in low serum (4\%) were transfected for 24-26 h, and serum starved for 10$14 \mathrm{~h}$ before lysis, to reduce background activity of mitotic kinases. Total transfection time was $34-40 \mathrm{~h}$.

\section{Western blot}

HEK293T cells were washed with ice-cold PBS, and lysed directly on the plate $(6 \mathrm{~cm})$ into lysis buffer $(500 \mu \mathrm{l}) ; 50 \mathrm{~mm}$ Tris $\mathrm{HCl}, 150 \mathrm{~mm} \mathrm{NaCl}, 1 \%$ NP-40, 1 mм EDTA, 1 mм DTT, 1 mм PMSF, $1 \times$ HALT protease/phosphatase inhibitor cocktail. Extra $\mathrm{NaF}$ and $\mathrm{NaOV}$ was added in the lysis buffer for phosphorylation experiments in addition to that already in the cocktail. After incubating lysates on a rotisserie at $4^{\circ} \mathrm{C}$ for $30 \mathrm{~min}$, lysates were cleared by centrifugation at $21,000 \times g$ for $15 \mathrm{~min}$ at $4^{\circ} \mathrm{C}$. We note that spinophilin (SPN) is not well solubilized under these lysis conditions, and we find that most of the SPN protein is in the pelleted material (data not shown). Supernatants were diluted to $1 \times$ with $5 \times$ Laemmli buffer for Western blot. Neuron cultures for Sema3a treatments were lysed directly in the 12-well plate into $80-100 \mu 11.5 \times$ Laemmli sample buffer for Western blot. Samples were vortexed, boiled, and spun at $18,000 \times g$ for $10 \mathrm{~min}$ before loading. For neurons, the protein equivalent to $100,000-150,000$ neurons was loaded per lane. Equivalent amounts of cells within each experiment were run on a $4 \%-20 \%$ polyacrylamide gel. After transfer onto nitrocellulose with the Bio-Rad Transblot Turbo, membrane was blocked with Li-Cor TBS blocking buffer for $1 \mathrm{~h}$ at room temperature. Primary and secondary antibodies were diluted in 50\% blocking buffer, 50\% TBS, final 0.1\% Tween-20. Primary antibodies were incubated overnight at $4^{\circ} \mathrm{C}$, and after washing, followed by near-infrared secondary antibodies for $1 \mathrm{~h}$ at room temperature. Blots were imaged with the dual-color Li-Cor Odyssey CLx imager, so each phosphoprobe could be directly compared with the total protein in the same band. Densitometry of Western blots was performed with Li-Cor Image Studio software, the same software used to acquire the fluorescent blot images. The phosphoantibody signal was divided by the total protein level (GFP tag or non-phospho-specific antibody), and normalized to control. Positions of molecular weight ladders (in $\mathrm{kD}$ ) are indicated next to the blot when there was room; otherwise, the value of the approximate position in $\mathrm{kD}$ is shown.

\section{Immunoprecipitation}

Cell lysates were prepared as though for Western blotting $500 \mu$ l of lysis buffer per $6 \mathrm{~cm}$ dish of confluent cells. After the $21,000 \times g$ spin, cells were precleared with $50 \mu \mathrm{l}$ of control agarose (Thermo Fisher Scientific) for 4-6 h. All incubations and washes were performed on the rotisserie at $4^{\circ} \mathrm{C}$. For immunoprecipitations of myc-tagged proteins, $12 \mu \mathrm{l}$ Bethyl 
goat anti-myc preconjugated beads were added to the precleared lysate overnight.

$\lambda$ phosphatase dephosphorylation experiments were prepared from transfected $10 \mathrm{~cm}$ dishes of confluents cells, and lysed with $1 \mathrm{ml}$ of lysis buffer (0.4\% NP-40) minus the phosphatase inhibitors in the GFP-DCX condition. Following clearing by centrifugation $(21,000 \times g, 15 \mathrm{~min})$, GFP-DCX lysates were split into two Eppendorfs, and to one, phosphatase inhibitors were added, and to the other 3200 units $(8 \mu \mathrm{l})$ of $\lambda$ phosphatase (SCBT) and a final concentration of $2 \mathrm{mM} \mathrm{MnCl}_{2}$. Both conditions were incubated at $30^{\circ} \mathrm{C}$ for $30 \mathrm{~min}$, and the $\lambda$ phosphatase-containing sample was quenched with EDTA and phosphatase inhibitors. Each sample was separately combined each with half of the nestin-myc lysate, and incubated overnight at $4^{\circ} \mathrm{C}$ with $12 \mu \mathrm{l}$ Bethyl goat anti-myc beads.

For immunoprecipitations of other proteins (nestin, dcx, p35), $2 \mu \mathrm{g}$ of the relevant antibody was added to the precleared lysate for $4-6 \mathrm{~h}$, followed by addition of $12 \mu \mathrm{l} \mathrm{A} / \mathrm{G}$ beads overnight. Experiments using protein $\mathrm{A} / \mathrm{G}$ beads for immunoprecipitation also used them for preclearing instead of the control agarose. After overnight incubation, all IPs were washed 6 times in $500 \mu \mathrm{l}$ IP buffer. For GFP nano-body precipitations for phosphorylation assays, to minimize time and thus loss of phosphoepitopes, no preclearing was performed, the GFP-trap beads were immediately added to post-21,000 $\times g$ spin lysates for $1 \mathrm{~h}$, and washed $2 \times$. All IPs were eluted by addition of $2 \times$ sample buffer and immediate Western blotting.

\section{Neuronal culture}

Primary cultures of cortical neurons were obtained from embryonic day 16 (E16) mouse cortex of either sex as described by Bott et al. (2019). The protocol follows institutional Animal Care and Use Committee protocols (University of Virginia, approved animal protocol \#3422). Cells were plated on acid-washed poly-L-lysine-coated coverslips and incubated with Neurobasal medium with 10\% FBS and penicillin-streptomycin. After $8 \mathrm{~h}$, the cells were transferred into serum and antibiotic-free neurobasal medium supplemented with B27 (Invitrogen) and Glutamax and cultured for a total of the indicated time periods (days in vitro). When WT versus $D c x$-null neurons were compared within an experiment, mice were dissected and cultures prepared at the same time for consistency of comparison.

For imaging purposes, cells were plated at $\sim 100,000$ cells per $15 \mathrm{~mm}$ coverslip or at 130,000/coverslip in electroporation experiments. Cortical neurons for culture were harvested at E16, just before gliogenesis. Thus, non-neuronal glia are rare in these cultures, but occasional nestin/Sox2-positive, GFAP-negative persisting neural stem cells were found. Cells were fixed at $24 \mathrm{~h}$ (for nontransfection experiments) or $42-$ $48 \mathrm{~h}$ for overexpression experiments.

For coimmunoprecipitation experiments in neurons, cells were prepared similarly as described above. Six million untransfected cells were plated per $6 \mathrm{~cm}$ dish. At $24 \mathrm{~h}$, the cells in the $6 \mathrm{~cm}$ dish were lysed and subjected to DCX immunoprecipitation as described above.

Cells for Sema3a treatments and Western blot, cells were prepared similarly as described above. Untransfected cells were plated at a density of 1 million cells per 12-well plate well. After $24 \mathrm{~h}$, cells were treated with mouse Sema3a (R\&D Systems) diluted in growth media for 5, 15, or $30 \mathrm{~min}$ at $1 \mathrm{~nm}$ as indicated. All conditions were subjected to $25 \mathrm{~nm}$ calyculin A treatment $3 \mathrm{~min}$ before lysis to inhibit relevant phosphatase activity before lysis. After treatments, media was removed thoroughly and cells were quickly lysed directly into $1.5 \times$ Laemmli sample buffer and subjected to Western blotting as described above. In some experiments as indicated, cells were pretreated with $10 \mu \mathrm{m}$ roscovitine (Cayman Chemical) for $30 \mathrm{~min}$ before Sema3a treatment.

\section{Neuron nucleofection}

After cells were dissociated from E16 mouse cortex, cells were electroporated by Amaxa $4 \mathrm{~d}$ nucleofection, according to the manufacturer's protocols; 800,000 cells were electroporated in the small volume $(20 \mu \mathrm{l})$ cuvettes using P3 primary cell solution and CL-133 program; $0.1 \mu \mathrm{g}$ of GFP plasmid (pCMV-GFP, Clontech) as a transfection marker, and $0.15 \mu \mathrm{g}$ of relevant myc-tagged construct was used in each nucleofection condition. The cells were then all plated in a $6 \mathrm{~cm}$ dish containing 6 poly-L-lysine-coated coverslips.

\section{Immunofluorescence}

Cells were fixed in prewarmed $4 \%$ paraformaldehyde-PHEM-sucrose (60 mм PIPES, 25 mм HEPES, 10 mм EGTA, 2 mм $\mathrm{MgCl}_{2}$ [PHEM], $0.12 \mathrm{M}$ sucrose, $4 \% \mathrm{PFA}, \mathrm{pH}$ 7.4) for preservation of the cytoskeleton and cellular morphology and fixed at room temperature for $16 \mathrm{~min}$. Thorough permeabilization was required for nestin/IF visualization in neurons. Coverslips were permeabilized with $0.25 \%$ Triton-X 100 in $1 \% \mathrm{BSA} / \mathrm{PBS}$ for $20 \mathrm{~min}$ and then blocked for $30 \mathrm{~min}$ in $10 \%$ BSA, $5 \%$ normal donkey serum in PBS all at room temperature. Primary antibodies were diluted in 1\% BSA in PBS, and secondary antibodies in $1 \%$ BSA, $0.5 \%$ normal donkey serum in PBS. Primary antibodies were incubated overnight at $4^{\circ} \mathrm{C}$, and secondary antibodies for $90 \mathrm{~min}$ at room temperature. Appropriate species-specific Alexa-350, -488, -568 , or -647-labeled secondary antibodies raised in Donkey (Invitrogen and Jackson ImmunoResearch Laboratories) were used for fluorescent labeling. Phalloidin-568 (Invitrogen) at 1:150 was used to visualize F-actin. Coverslips were mounted with ProLong Gold (Invitrogen). Wide-field images for quantification were captured with a Z1-Observer with a $40 \times$ objective (Carl Zeiss). Images were captured with the Axiocam503 camera using Zen software (Carl Zeiss) and processed identically within experiments. Confocal images of the whole neurons were captured with inverted LSM880 confocal microscope (Carl Zeiss) using a $63 \times$ objective. Enhanced resolution images of GCs were captured using a Nikon TiE with A1 confocal microscope with a $100 \times$ objective and NA 1.49. Images were deconvolved using Landweber for 10 iterations. No nonlinear image adjustments were performed.

In utero electroporation (IUE) with CRISPR

The IUE CRISPR/Cas9 strategy is as follows: a gRNA sequence targeting the C-terminus of the Actb coding sequence (5'-AGTCCGCCT AGAAGCACTTG) was cloned into eSpCas9Opt1.1, expressing enhanced-specificity Cas9 and using an optimized gRNA scaffold. To construct Actb_3xHA, tandem HA tag sequences were cloned between two Actb gRNA recognition sequences in the same orientation. Timedpregnant dams were anesthetized with ketamine $(100 \mathrm{mg} / \mathrm{kg})$ and xylazine $(10 \mathrm{mg} / \mathrm{kg})$ via intraperitoneal injection, and given carprofen $(5 \mathrm{mg} / \mathrm{kg})$ via subcutaneous injection for analgesia. Uterine horns of an E13.5 pregnant CD-1 dam were exposed by midline incision. Lateral ventricles of embryos were injected with plasmid mix, composed of eSpCas9opt1.1-Actb_gRNA $(1 \mu \mathrm{g} / \mu \mathrm{l})$, Actb_3xHA $(1.5 \mu \mathrm{g} /$ $\mu \mathrm{l})$, and CAG-Lifeact3xBFP $(1 \mu \mathrm{g} / \mu \mathrm{l})$, and $0.1 \%$ Fast Green FCF to visualize solution during intraventricular injection. Successful genome editing and repair in a subset of transfected cells yielded an HAtagged Actb locus and led to sparse expression of ACTB-3xHA in individual cells. Electroporation paddles were positioned on opposing sides of the ventricle and 4-5 pulses of $27 \mathrm{~V}$ (45 ms each) were delivered, with $950 \mathrm{~ms}$ between pulses, delivered using a BTX Harvard Apparatus ECM 830 power supply. Brains were isolated $3 \mathrm{~d}$ after electroporation, at EXVI.5, and fixed overnight in 4\% PFA. These experiments were conducted in compliance with ethical regulations for animal research. The study protocol was reviewed and approved by the University of Michigan Institutional Animal Care \& Use Committee.

\section{Histology}

Fixed in utero electroporated E16.5 mouse brains were then washed with PBS, embedded in agarose, and vibratome sectioned into $100 \mu \mathrm{m} \mathrm{sec}-$ tions. Sections were stained as floating sections, permeabilized, and blocked with $3 \%$ normal donkey serum $+0.3 \%$ Triton-X 100 in PBS for $1 \mathrm{~h}$ at room temperature. Antibodies were diluted in the same permeabilization buffer with indicated primary and secondary antibodies sequentially overnight at $4^{\circ} \mathrm{C}$. Sections were thoroughly washed over a period of $10 \mathrm{~h}$ at room temperature with $0.5 \%$ BSA and $0.1 \%$ Triton-X 100 in PBS after primary and secondary antibody incubations. Sections were mounted on slides with Prolong Gold using $22 \times 22 \mathrm{~mm} 1.5$ Fisher brand coverslips. Confocal and Airyscan imaging of cryosections was conducted on an inverted LSM880 confocal microscope (Carl Zeiss), 
available in the University of Virginia microscopy core facility using a $63 \times$ objective.

\section{Molecular biology}

\section{Plasmids}

Plasmids are as follows: GFP-DCX (Yap et al., 2016); negative control substrates, GFP tagged: DCLK1 (Shin et al., 2013); CRMP2A (Balastik et al., 2015); FAK (Addgene); Drebrin-YFP (Addgene); Tau-YFP (George Bloom); P35 (Addgene); HA-CDK5 (Addgene); HA-CDK5-DN (D145N) (Addgene); Myc-SPN Lawrence Brass (UPenn)(Yap et al., 2016); INA-myc (Sino Biological); and Nestin-myc (via original cDNA Bruce Lahn) (Bott et al., 2019) PCR was used to generate EcoRI and HindII sites on the $5^{\prime}$ or $3^{\prime \prime}$ end, respectively, of full-length mouse nestin. Both the insert and vector were treated with EcoRI and HindII, the cut insert was ligated into pCDNA3.1 Myc-His(+) B; Myc EV 3.1 B (Stratagene); GFP (Clontech); and CMV-HA EV (Addgene). New constructs made for this study are as follows: nestin mutants: Nestin-myc T316A-We used traditional primer mutagenesis for this plasmid derived from the pcDNA 3.1 B Nestin(wt)-myc plasmid. Only one nucleotide change was needed to mutate $\mathrm{T}(946 \mathrm{ACA})$ to $\mathrm{A}(946 \mathrm{GCA})$. Primer: 5'-gaacttcttccaggtgcctgcaagcgagagttc-3'. Nestin-myc T316D could not be obtained via traditional mutagenesis and was made, instead, via gene synthesis from genscript of a large $>1 \mathrm{~kb}$ piece of nestin that included the three nucleotide changes: $\mathrm{T}(946 \mathrm{ACA})$ to $\mathrm{D}(946 \mathrm{GAT})$. The 5 ' end had a HINDIII site for incorporation into the MCS of pcDNA 3.1 $B$ nestin(wt)-myc plasmid. An EcoR1 site present in WT nestin was used for the $3^{\prime \prime}$ end.

\section{Image analysis}

All image analyses were performed on ImageJ blinded to experimental conditions. Transfected neurons were identified by having a prominent GFP fluorescence. Stage 3 neurons were identified (according to Banker staging) by having one neurite (the future axon) at least $2 \times$ longer than the next longest neurite. The distal portion of the future axon was analyzed for morphologic measurements as the "axon GC." Images were analyzed as in Bott et al. (2019).

Filopodial protrusions. Filopodial protrusions were counted using the phalloidin channel, and any protrusions from the GC were counted.

Axon length. Axon length was the length of the axon from the cell body to the GC tip as in Bott et al. (2019).

GC area. GC area was assessed by measuring traced outlines in the GC, not counting the filopodial protrusions, and extending down proximally to the point where the splayed GC MTs consolidate into bundles in axon shaft as in Bott et al. (2019).

Transfected $\operatorname{Cos} 7$ cells. Pearson correlation coefficients were determined using the Coloc module in Imaris 9.5.1. ROIs were defined as the entire area within a transfected cell expressing the two markers of interest; then the Pearson value was determined within that ROI (termed "Pearson's coefficient in the ROI volume" in Imaris).

\section{Statistical analysis}

All data were statistically analyzed using Prism software. Datasets were first evaluated for normality by using the Shapiro-Wilk normality test. This result was used to decide whether parametric or nonparametric tests were to be used. When more than one comparison was made, the corresponding ANOVA test was used. When only two conditions were compared, we used a $t$ test. Figure legends specify details; for each set of experiments, we made an effort to show precise $p$ values when shown. $p$ values reaching statistical significance of at least $<0.05$ are in bold in the figures.

\section{Microscope image acquisition}

Wide-field images for quantification were captured with a Z1-Observer with a $40 \times$ objective (Carl Zeiss). Images were captured with the Axiocam503 camera using Zen software (Carl Zeiss) and processed identically within experiments.

Confocal images of the whole neurons or brain sections were captured with inverted LSM880 confocal microscope (Carl Zeiss) using a
$63 \times$ objective with Zen software. Airyscan was used for some brain section imaging.

Enhanced resolution images of GCs were captured using a Nikon TiE with A1 confocal microscope with a $100 \times$ objective and NA 1.49. Images were deconvolved using Landweber for 10 iterations. No nonlinear image adjustments were performed.

\section{Reagents}

Antibodies. Antibodies are as follows: mouse anti-Nestin 2Q178, 1:200 IF, 1:500 IHC, 1:500 WB, Santa Cruz Biotechnology, catalog \#sc-58 813, RRID:AB_784786; goat anti-Nestin R-20: raised against peptide in the unique C-terminal tail region of nestin 1:200 IHC, Santa Cruz Biotechnology, catalog \#sc-21 249, RRID:AB_2267112; rabbit anti-DCX, 1:1200 IF, IHC, 1:4000 WB, Abcam, catalog \#ab18723, RRID:AB_ 732011; mouse anti- $\beta$ III tubulin Tuj1, 1:800 IF, a generous gift from the Deppman Lab University of Virginia A. Frankfurter, University of Virginia (Department of Biology, catalog \#TuJ1, $\beta$ III tubulin, RRID: AB_2315517); chicken anti- $\beta$ III tubulin, 1:200 IF, Aves Labs, catalog \#TUJ, RRID:AB_2313564; rat anti $\alpha$-tubulin, 1:2000 WB, Santa Cruz Biotechnology, catalog \#sc-53030, RRID:AB_2272440; rabbit antivimentin, 1:100 IF, 1:100 IHC, 1:1000 WB, Bioss, catalog \#bs-0756R, RRID:AB_10855343; rabbit anti-vimentin, 1:1000 IF, Abcam, catalog \#ab92547, RRID:AB_10562134; mouse anti-Myc tag 9E10, 1:1000 WB, 1:200 IF, Santa Cruz Biotechnology, catalog \#sc-40, RRID:AB_627268; rat anti-Myc tag 9E1, 1:1000 WB, ChromoTek, catalog \#9e1-100, RRID: AB_2631398; rat anti-HA, 1:2000 WB, ChromoTek, catalog \#7c9, RRID: AB_2631399; rabbit anti-GFP D5.1, 1:5000 WB, Cell Signaling Technology, catalog \#2956, RRID:AB_1196615; mouse anti-GFP N86/8, 1:5000 WB, University of California Davis/National Institutes of Health, NeuroMab facility, catalog \#73-131, RRID:AB_10671444; rabbit antiCDK substrate multiMab, 1:1000 WB, Cell Signaling Technology, catalog \#9477, RRID:AB_2714143; rabbit anti-pS297 DCX, 1:1000 WB, Cell Signaling Technology, catalog \#4605, RRID:AB_823682; rabbit antipS334 DCX, 1:2000 WB, Cell Signaling Technology, catalog \#3453, RRID:AB_2088485; mouse anti-CDK5, 1:2000 WB, 1H3, Cell Signaling Technology, catalog \#12134, RRID:AB_2797826; rabbit anti-p35/p25, 1:2000 WB, C64B10, Cell Signaling Technology, catalog \#2680, RRID: AB_1078214; mouse anti-SPN (Neurabin II), 1:2000 WB, Santa Cruz Biotechnology, catalog \#sc-373974, RRID:AB_10918794; mouse antiDrebrin, 1:2000 WB, Santa Cruz Biotechnology, catalog \#sc-374269, RRID:AB_10990108; mouse anti-Phf1 tau-supernatant, 1:50 WB, P. Davies Albert Einstein College of Medicine (New York), catalog \#PHF1, RRID:AB_2315150 (a gift from George Bloom); rabbit anti-pS522 Crmp2a, 1:1000 WB, Thermo Fisher Scientific, catalog \#PA5-37550, RRID:AB_2554159; mouse anti-pS732 FAK, 1:1000 WB, Santa Cruz Biotechnology, catalog \#sc-81 493, RRID:AB_1125825; rabbit antipY397 FAK, 1:1000 WB, Bioss, catalog \#bs-1642R, RRID:AB_10856939; Gt anti-myc agarose, 1:50 IP, Bethyl, catalog \#S190-104, RRID:AB 66618; GFP trap agarose, 1:100 IP, ChromoTek, catalog \#gta-20, RRID: AB_2631357; GFP booster nanobody, 1:600 IF, ChromoTek, catalog \#gba488-100, RRID:AB_2631434; and phalloidin 568, 1:150 (Invitrogen).

Cell lines. Cell lines are as follows: Hek293 cell lines, ATCC, catalog \#PTA-4488, RRID:CVCL_0045; and Cos7 cell lines, ATCC, catalog \#CRL-1651, RRID:CVCL_0224.

\section{Results}

\section{Nestin selectively augments cdk5 phosphorylation of DCX, but not of other cdk5 substrates}

Our recent work (Bott et al., 2019) discovered an unexpected role for nestin in axonal GCs: downregulation of nestin increases GC size and decreases Sema3a sensitivity. In other cell types, nestin has been shown to affect the activity of cdk5. In neuromuscular junction development, for example, nestin deletion phenocopies cdk5 deletion or treatment with the cdk5 inhibitor roscovitine by altering levels and localization of cdk5 activity (Lin et al., 2005; Yang et al., 2011). Therefore, we hypothesized that nestin acted via cdk5 in early neurons. Cdk5 is important 
for many processes in neurite outgrowth, including responses to guidance cues (Dhavan and Tsai, 2001; Kawauchi, 2014). KO mouse models that lack $c d k 5$ produce a cortex with disorganized layering, similar to what is seen in lissencephaly (Tanaka et al., 2004), and result in perinatal lethality (Ohshima et al., 1996; Hirasawa et al., 2004). Other studies have shown that balancing cdk5 activity is important, as increasing cdk5 function proves also disruptive (Cheung and Ip, 2012). Since nestin binds the cdk5 activator p35 (Sahlgren et al., 2003, 2006; Yang et al., 2011), and cdk5 inhibition abrogates the effect of nestin-associated Sema3a-mediated GC collapse in neurons (Bott et al., 2019), we reasoned that nestin might change the phosphorylation of one or more cdk5 substrates to mediate the nestin-dependent changes in axonal GCs. We thus tested whether the phosphorylation of several known neuronal cdk5 substrates was affected by the expression of nestin.

To this end, we used an "in-cell" phosphorylation assay in transfected HEK293 cells (Fig. 1A-E). HEK293 cells endogenously express cdk5, but not the cdk5 activator p35 (see Fig. $2 A$, $\mathrm{cdk} 5$ and p35 immunoblot). They thus have minimal cdk5 activity under basal conditions. We focused on Focal Adhesion Kinase (FAK), Collapsin Response Mediator Protein 2 (Crmp2), tau, and DCX since they are known to be phosphorylated in neurons downstream of Sema3a signaling and phospho-specific antibodies are commercially available (Fig. $1 A-E$ ). Transfection of HEK293 cells with p35 to activate cdk5 led to increases in phosphorylation of GFP-tagged FAK, Crmp2, or tau, as expected (Fig. $1 A, C, D$ ). Surprisingly, p35 expression was not sufficient to increase cdk5-mediated phosphorylation of DCX in this context (Fig. 1E). When nestin was expressed in addition to p35, FAK, Crmp2, or tau showed no further increase in phosphorylation. However, DCX phosphorylation became significantly increased when nestin was coexpressed with p35. A myc-EV was used as a negative control. For quantification of all conditions, we used ratios of the phospho-protein signal to total expression of the GFP-tagged substrate protein (Fig. $1 A^{\prime}-E^{\prime}$ ). As an additional control, we used an antibody to a phospho-tyrosine residue on FAK. As expected, pY397-FAK was not affected in any condition (Fig. $1 B, B^{\prime}$ ). We note that there was basal phosphorylation for some of these substrates, even in the absence of overexpressed p35, which is likely due to activity of other kinases in HEK293 cells. We thus discovered a surprising substrate selectivity for nestin-enhanced phosphorylation by $\mathrm{p} 35 / \mathrm{cdk} 5$. This finding suggested the hypothesis that DCX could be a downstream effector for the nestin-dependent effects seen in the GCs of young neurons.

\section{Nestin, but not INA, promotes phosphorylation of DCX by cdk5}

In order to more fully characterize the effect of nestin on DCX phosphorylation, we repeated the phosphorylation assay of DCX with additional controls. As shown above, we saw a significant increase in pS297-DCX levels only when p35 and nestin are both coexpressed (Fig. $2 A, A^{\prime}$, gray bars). In contrast to the phosphorylation of S297, the phosphorylation of S334 by JNK was not changed by coexpression of nestin (Fig. $2 A, A^{\prime}$, red bars). In order to test the specificity of the phospho-specific antibodies, we expressed SPN (Fig. $2 A, A^{\prime}$ ), which is a known protein phosphatase 1 (PP1) scaffold and promotes dephosphorylation of both the cdk5 and JNK sites of DCX (Shmueli et al., 2006; Tsukada et al., 2006; Bielas et al., 2007). The levels of both pS297-DCX and pS334-DCX were greatly diminished by SPN coexpression. We note that nestin coexpression with p35 consistently resulted in

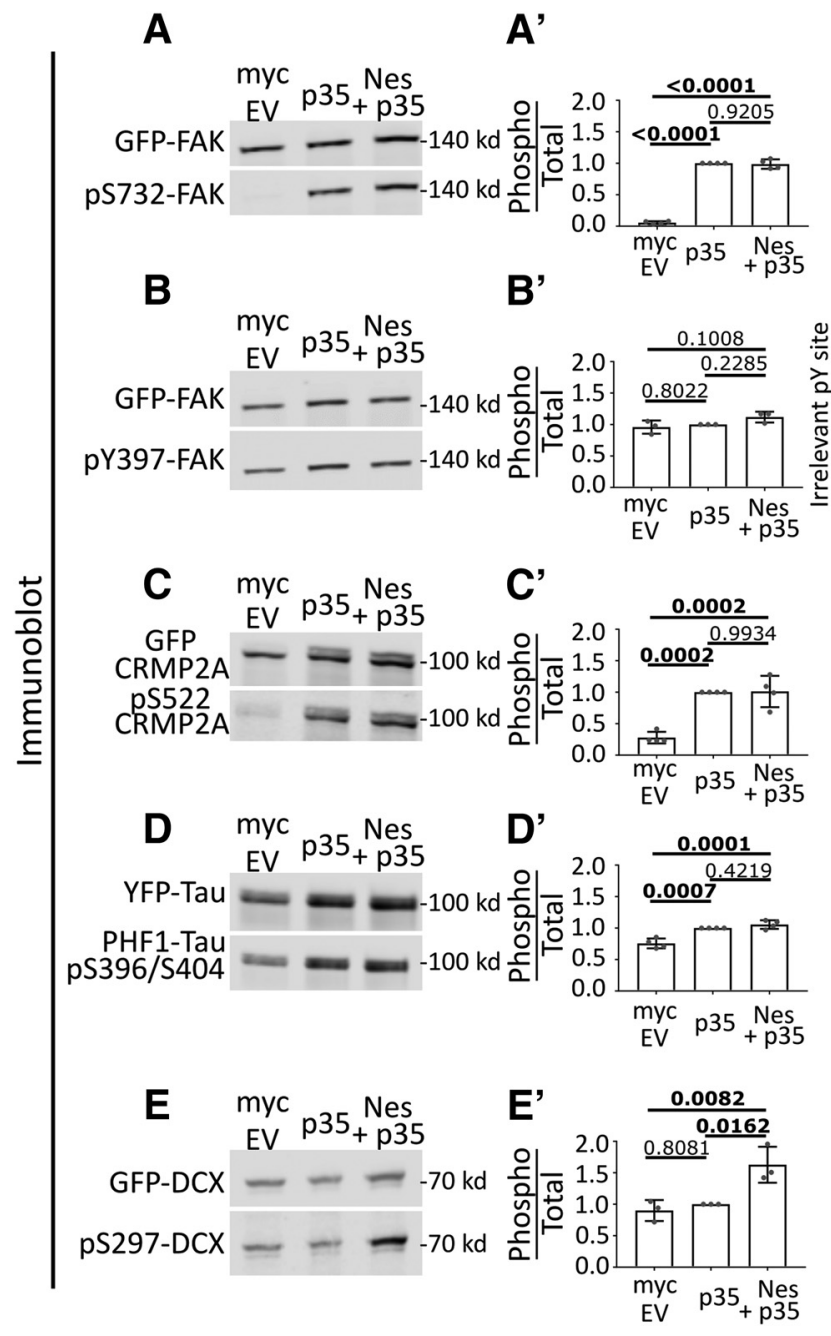

Figure 1. Nestin selectively augments $c d k 5$ phosphorylation of $D C X$, but not other $c d k 5$ substrates. $\boldsymbol{A}-\boldsymbol{E}$, Comparison of cdk5-mediated phosphorylation in HEK293 cells of several cdk5 substrates in the indicated transfection conditions: Myc-EV (left lane), Myc-EV + p35 (middle lane), Nes-myc + p35 (right lane). Cdk5-dependent phosphosites on FAK $(\boldsymbol{A})$, Crmp2 $(\boldsymbol{C})$, tau $(\boldsymbol{D})$, and DCX $(\boldsymbol{E})$ were assessed for p35 and nestin dependence. The pY397 site on FAK $(\boldsymbol{B})$ is a negative control because $\mathrm{cdk} 5$ is a Ser/Thr kinase. $\boldsymbol{A}^{\prime}-\boldsymbol{E}^{\prime}$, The level of phosphorylation of each substrate was quantified by densitometry analysis of the phosphosignal compared with total (GFP) and graphed to the right of each blot. Error bars indicate SD. The statistical test used was one-way ANOVA with Tukey's correction. $N=3$ or 4 independent experiments. $p$ values of $<0.05$ are indicated in bold.

an increase in total DCX levels. Again, we used the ratio of the phospho-DCX signal to total expression of GFP-DCX to normalize for input levels of DCX.

We next conducted several controls to ascertain that the increased phosphorylation of DCX was attributable to cdk5 activity and not to other endogenous kinases present in HEK293 cells. The cdk5 inhibitor roscovitine reduced pS297-DCX phosphorylation, but neither SP600125 (a JNK inhibitor) nor Ro3306 (a cdk1/cdc2 inhibitor with minimal cross-reactivity with cdk5) changed the pS297-DCX levels (Fig. $2 B, B^{\prime}$ ), supporting previous literature that S297 is a cdk5 site on DCX (Tanaka et al., 2004). Multiple residues in addition to S297 are phosphorylated by cdk5 on DCX, but specific antibodies to detect these phosphorylation events are not available. We obtained another antibody, which recognizes the general "K/H pSP" phospho-epitope consensus site for CDKs to confirm our results with the pS297DCX antibody. GFP-DCX was precipitated from lysates with 
A

Myc EV Nestin SPN INA

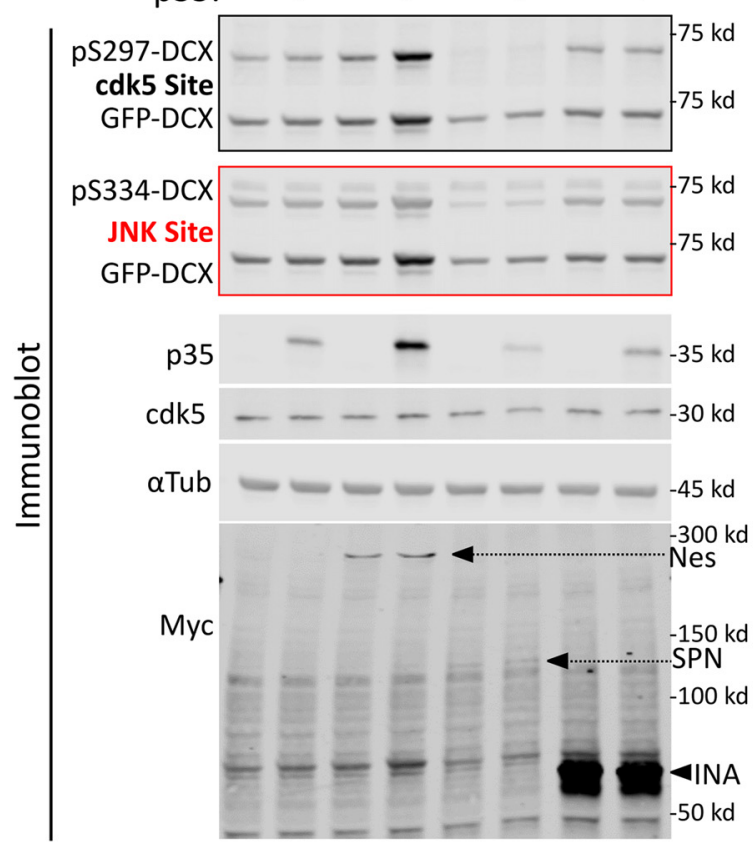

C

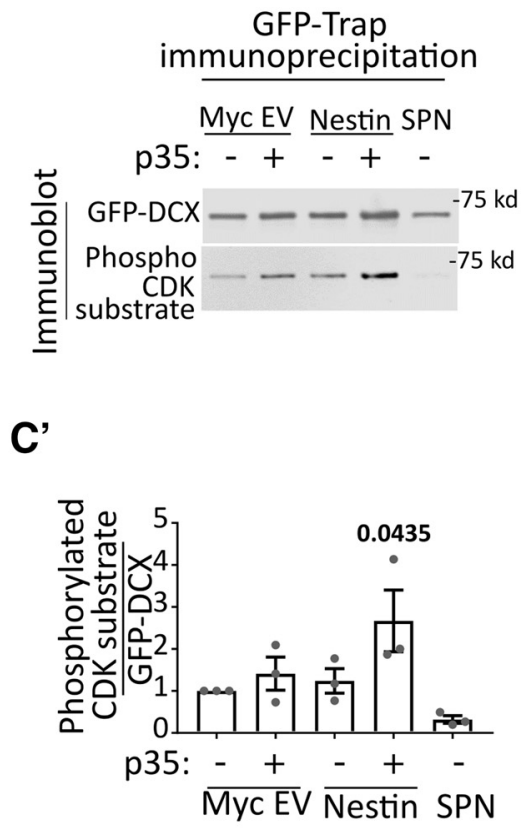

$A^{\prime}$

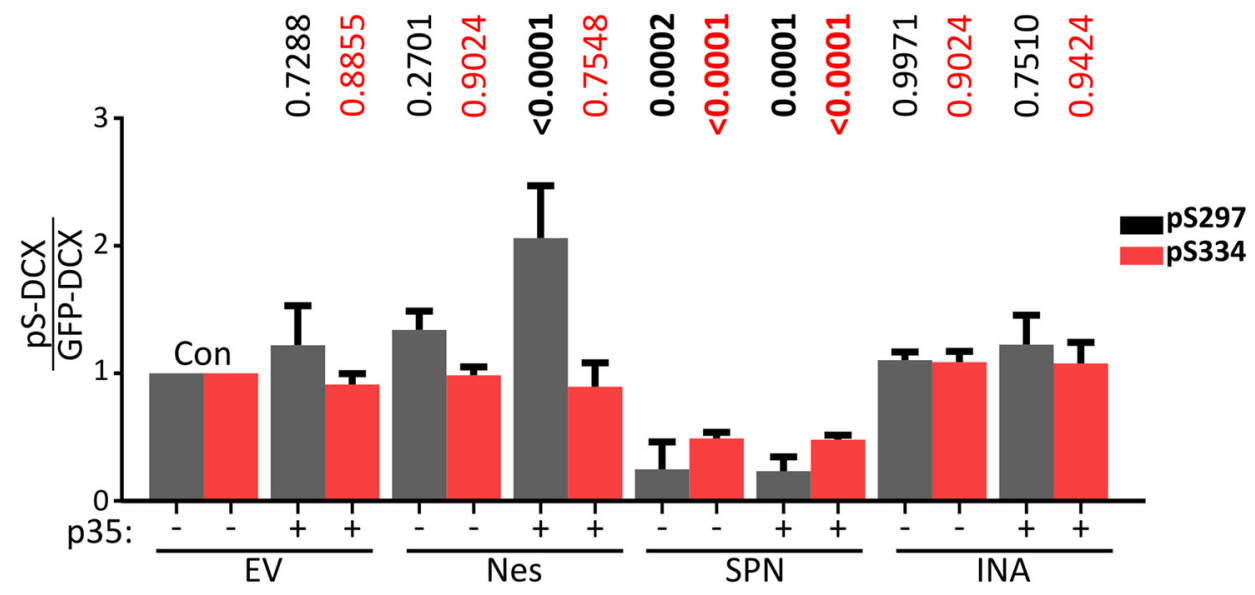

B

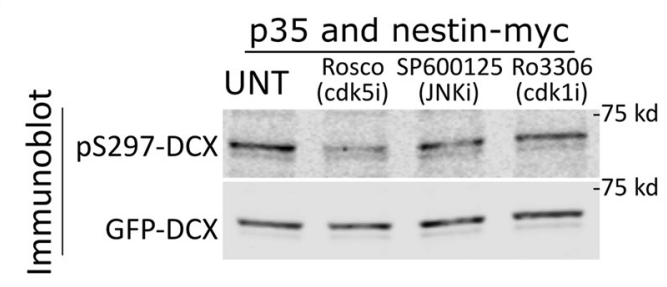

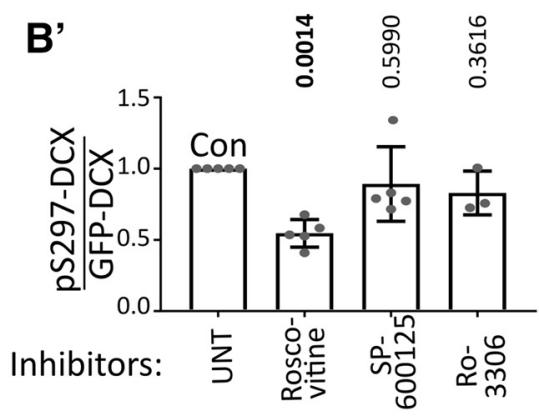

Figure 2. Nestin, but not INA, promotes phosphorylation of DCX by cdk5. A, Myc EV, Nestin-myc, INA-myc, or myc-SPN was expressed in HEK293 cells with DCX alone (-) or with both p35 and DCX $(+)$. Cell lysates were run on two replicate Western blots and both blotted against GFP for total levels of GFP-DCX. Each was blotted with the relevant phospho-specific antibodies to pS297-DCX (a cdk5 site) or pS334-DCX (a JNK site). The levels/presence of relevant proteins are shown, including ckd5 (endogenous), p35 (overexpressed), $\alpha$-tubulin (endogenous, loading control), and the overexpressed myc-tagged constructs. Arrows point to the relevant myc-tagged protein bands. $\boldsymbol{A}^{\prime}$, Quantification of $\boldsymbol{A}$ by densitometry. Ratio of phospho-DCX to total DCX are plotted. Black bars represent pS297-DCX/total DCX. Red bars represent pS334-DCX/total DCX. Colors represent the outline around the relevant blot. Error bars indicate SD. $N=4-6$ independent experiments. Every condition was compared with every other condition with an ordinary one-way ANOVA test with Tukey's correction. $p$ values compared pairwise to control (CON) are shown. Additional comparisons not shown are pS297 level. Nestin + p35 condition is significantly different from p35 alone $(p<0.0001)$ and nestin alone $(p=0.0004)$, whereas pS334 is not significantly different ( $>0.9999$ and 0.8979 , respectively) for the same comparisons. $p$ values of $<0.05$ are indicated in bold. The values for DCX phosphorylation are from additional independent 
GFP-trap agarose beads to separate DCX from other reactive endogenous cdk substrates in this experiment. Similar to our results using the anti-pS297DCX antibody, a significant nestin-dependent increase in cdk5 phosphorylation of DCX could also be seen with this cdk phospho-substrate antibody (Fig. 2C; and quantified in Fig. $2 C^{\prime}$ ). Again, expression of SPN to recruit PP1 to DCX abolished the band on Western blot.

Last, we tested a different neuronal IF protein, INA, which is not known to bind to p35. INA did not promote DCX phosphorylation by $\mathrm{p} 35 / \mathrm{cdk} 5$, arguing for a specific enhancement of DCX phosphorylation by nestin (Fig. $2 A, A^{\prime}$ ).

\section{Nestin forms a complex specifically with DCX}

Next, we asked by what mechanism nestin might selectively enhance cdk5 phosphorylation of a specific cdk5 substrate. We first tested whether nestin formed a complex with any of the cdk5 substrates. We cotransfected Nestin-myc or myc-EV (EVnegative control) with several GFP-tagged cdk5 substrates (DCX, DCX-like-kinase 1 [DCLK1], CRMP2, Tau, Drebrin, and FAK) individually in HEK293 cells, followed by cell lysis and anti-myc tag immunoprecipitation (Fig. 3A). DCX, CRMP2, Tau, Drebrin, and FAK are cdk5 substrate proteins known to be downstream of Sema3a. We used DCLK1 as an additional control since it shares sequence homology with DCX, and is a cdk5 substrate (Contreras-Vallejos et al., 2014). The efficiency of coimmunoprecipitation with nestin-myc was quantified for each GFP-tagged substrate by densitometry analysis as a ratio of immunoprecipitated protein to the total amount of protein in the corresponding input. Low levels of other substrates, such as DCLK1 and CRMP2, were also seen in the coimmunoprecipitations, but not at significant levels relative to the EV control. Only GFP-DCX significantly coimmunoprecipitated with nestin-myc (Fig. $3 A, A^{\prime}$ ).

To determine whether the nestin-DCX complex occurred endogenously in the relevant tissue, lysates from embryonic rodent brain were subjected to IP with either anti-nestin (Fig. $3 B$ ) or anti-DCX (Fig. $3 B^{\prime}$ ) antibodies and compared with nonimmune IgG. An endogenous complex of nestin and DCX was recovered for both IPs. IP with anti-nestin antibody resulted in efficient coimmunoprecipitation of DCX. In contrast, relatively low amounts of nestin were recovered in the IP with an antiDCX antibody; but compared with the known DCX interacting protein SPN (a positive control), similar levels of coimmunoprecipitation efficiency were detected. This low efficiency is likely due to the fact that DCX has a multitude of binding interactions

$\leftarrow$

experiments separate from those shown in Figure 1E. B, HEK293 cells transfected simultaneously with p35, nestin-myc, and GFP-DCX were treated with inhibitors of cdk5 (roscovitine), JNK (SP600125), cdk1 (Ro2206), or vehicle (UNT) for $6 \mathrm{~h}$ before lysis. Only roscovitine significantly decreased levels of pS297-DCX. $\boldsymbol{B}^{\prime}$, Quantification of the blots in $\boldsymbol{B}$. Error bars indicate SD. Ordinary one-way ANOVA with Dunnett's correction was performed. Pairwise $p$ values are shown for each condition compared with untreated control (UNT). $N=3-5$ independent experiments. $p$ values of $<0.05$ are indicated in bold. C, HEK293 cells were transfected similarly to Figure $2 A$, and total cdk5-mediated phosphorylation of GFP-DCX was assessed using an antibody to a generic K/H SP cdk5 phosphorylation motif to detect. Because many proteins in HEK293 cell lysates can be recognized with this antibody, cell lysates were subjected to GFP-trap incubation and precipitation to isolate GFP-tagged DCX from other reactive proteins and subsequently subjected to Western blot. $\boldsymbol{C}^{\prime}$, Densitometry quantification of $\boldsymbol{C}$ where levels of signal to the phospho-reactive antibody is ratioed to the level of total DCX. Error bars indicate SD. A one-way ANOVA with Dunnett's correction was used to compare each condition to the control cells transfected with GFP-DCX only. $N=3$ independent experiments. $p$ values of $<0.05$ are indicated in bold. which might be mutually exclusive. The noninteracting protein drebrin was not detectable in the coimmunoprecipitation, serving as a negative control. In agreement with these findings, we also found nestin by mass spectrometry in DCX IPs of embryonic rat brain (C.C.Y., unpublished data).

Since brain contains multiple cell types, we repeated the IP with lysates from E16 cortical neurons cultured for 1 DIV. Indeed, nestin coimmunoprecipitated with DCX (Fig. 3C) in DIV1 cortical cultures. As a negative control, we conducted IPs from neuronal cultures prepared from $D c x$ KO E16 cortex. The $D c x$ KO neurons expressed similar levels of nestin as WT cultures, but no nestin was detected in the DCX IP (Fig. $3 C$ ). We thus discovered a novel endogenous complex of DCX and nestin in immature cortical neurons.

Since DCX is MT-associated and nestin is an IF, we wondered where a complex of nestin with DCX would localize in cells. In order to test whether DCX and nestin affected their respective cellular localizations, we transfected Cos7 cells, which are large, well-spread cells which have spatially resolvable MT and vimentin IF filament systems. As shown previously by us and others, GFP-DCX decorates MTs (Gleeson et al., 1999; Sapir et al., 2000; Tsukada et al., 2005; Ge et al., 2006; Moslehi et al., 2017; Yap et al., 2018, 2012), but it is not colocalized with vimentin (Fig. 4A). Conversely, Nes-myc colocalized with endogenous vimentin in the absence of DCX (Fig. 4B). When DCX was coexpressed, nestin became enriched on DCX-decorated MT bundles (Fig. 4C). When a DCX-related MAP, GFP-DCLK1, was expressed instead of DCX, nestin remained associated with vimentin IFs (Fig. 4D). Other IFs also expressed in early neurons, such as INA, did not enrich with DCX-MT bundles (Fig. 4E). Pearson's coefficient of colocalization was quantified for double-transfected Cos7 cells, demonstrating strong colocalization between DCX and nestin, and significantly less colocalization between DCLK1 and nestin, or DCX and INA (Fig. 4F). Nestin can thus be recruited to DCXdecorated MTs.

\section{Nestin and DCX colocalize in the distal axon of cortical neurons in vitro and in vivo}

We previously described a striking localization of nestin in the distal axon of cultured E16 mouse cortical neurons at 1 DIV that is rapidly lost as neurons matured (Bott et al., 2019), and nestin mRNA has recently been found to be enriched in neurites (von Kügelgen and Chekulaeva, 2020). DCX is enriched in the distal axon but is also present in dendrites (Friocourt et al., 2003; Schaar et al., 2004). Simultaneous staining with antibodies against DCX and nestin revealed colocalization in the distal axon (Fig. 5A, arrowhead), but not in dendrites. Enhanced resolution microscopy of the distal axon showed that nestin codistributed with DCX in the "wrist" region of the axonal GC (Fig. 5B) and appeared to be filamentous. Nestin staining also extended past the "wrist" into the central domain of the GC. Interestingly, we observed occasional nestin staining in some of the proximal filopodia of the GC (Fig. 5B, arrowhead), which were also stained for DCX.

We previously showed nestin immunostaining in E16.5 mouse cortex in axon fascicles in the intermediate zone (IZ), which are interwoven among highly expressing radial glial processes (Bott et al., 2019). However, we were unable to discern single axons in vivo, let alone single GCs, due to the high density of axons in the IZ. In order to visualize nestin localization in individual developing axons in vivo, we used IUE to sparsely label individual neurons (Fig. 5C). Using CRISPR/Cas9 genome editing, $3 \mathrm{xHA}$ tags were introduced into the endogenous $\beta$-actin 

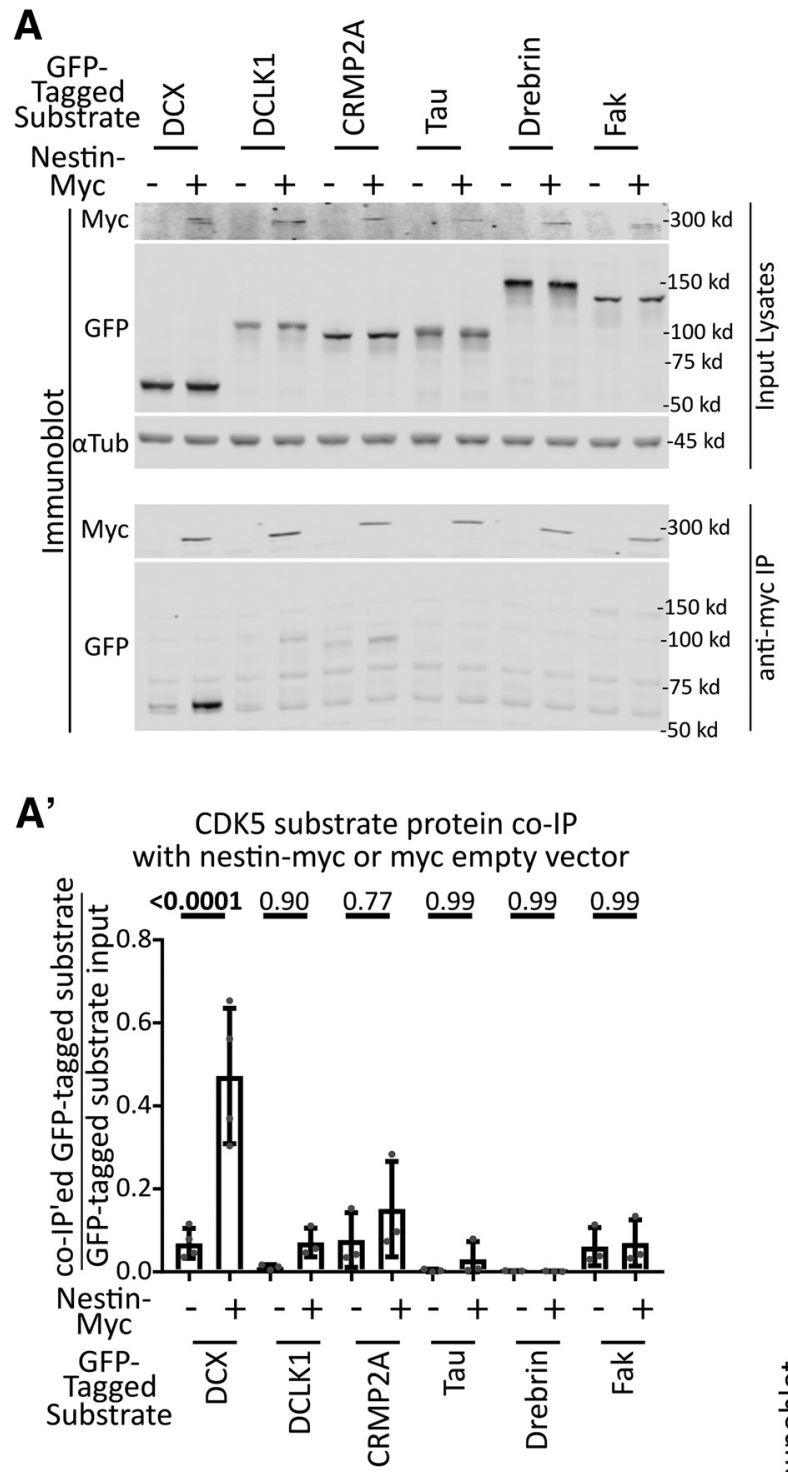

B

\section{E17 rat brain}
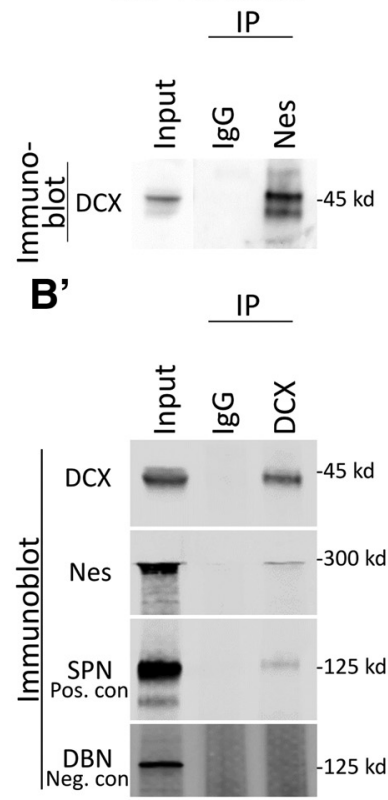

C

E16 Mouse

Cortical neuron culture

1 DIV

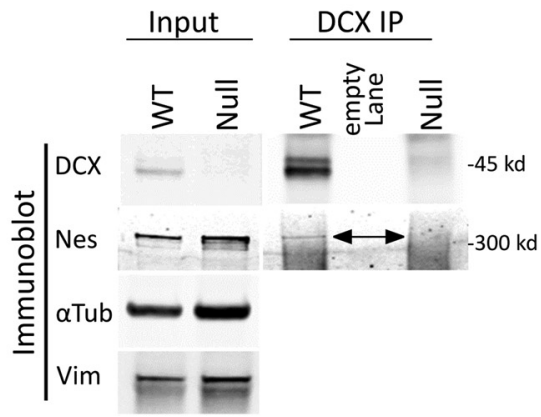

Figure 3. Nestin forms a complex specifically with DCX. A, Candidate screen for cdk5 substrates that interact with nestin by coimmunoprecipitation. Six different GFP-tagged cdk5 substrates were expressed with or without nestin-myc in HEK293 cells and subjected to immunoprecipitation with anti-myc antibody. An empty vector (EV) was used as a negative control. Top, Input lysates are shown. $\alpha$-Tubulin is used as a loading control. Bottom, Anti-myc IPs are probed against myc or GFP. Only GFP-DCX coimmunoprecipitates with nestin-myc. $A^{\prime}$, Densitometry analysis of each coimmunoprecipitated substrate relative to its input. Statistical comparisons (ordinary one-way ANOVA with Sidak's correction) were performed between the EV myc IP compared with nestin-myc IP. Errors bars indicate SD. $n=$ at least 3 for each condition. $p$ values are indicated above the graph. $p$ values of $<0.05$ are indicated in bold. $\boldsymbol{B}, \boldsymbol{B}^{\prime}, \mathbb{I P}$ of endogenous nestin and $D C X$ complexes from brain. $\boldsymbol{B}, D C X$ coimmunoprecipitation with anti-nestin antibody from E17 rat brain compared with a control IgG. $\boldsymbol{B}^{\prime}$, Nestin coimmunoprecipitation with anti-DCX antibody from E17 rat brain compared with a control lgG. As a positive control, DCX IPs were probed against SPN. Drebrin serves as a negative control. C, Anti-DCX immunoprecipitation from 1 DIV cortical EXVI mouse neurons results in coimmunoprecipitation of nestin from WT neurons, but not from Dcx KO (null) neurons, demonstrating specificity of the IP. The double-headed arrow points to the band corresponding to nestin. No anti-DCX immunoreactivity is seen in the Western blots of DCX-null neurons compared with WT, also demonstrating specificity of the antibody. $\alpha$-Tubulin and vimentin immunoblots are shown as loading controls.

locus, which enabled us to label the actin cytoskeleton of developing neurons, including the GC (Fig. 5F, diagram). Three days after in utero electroporation (IUE), brains were fixed, sectioned, and immunostained for the HA-tag in combination with staining for DCX and nestin (Fig. 5C). This technique introduced the HA tag into a small number of newly born neurons, which were scattered throughout the IZ and cortical plate (CP) at multiple stages of maturation. We expected to find nestin-positive axon tips in only a subset of labeled neurons (Bott et al., 2019) and therefore focused on neurons that were migrating though the IZ and had not yet reached the $\mathrm{CP}$ (Fig. 5C, white asterisk). These neurons are less mature and still in the early stage of axon extension (Namba et al., 2014; Sakakibara and Hatanaka, 2015) and thus most analogous to the young neurons we used (and quantified by Bott et al., 2019) in culture. One such neuron (soma marked with red arrowhead, axon tip marked with white arrowhead) is shown enlarged in Figure $5 C^{\prime}$. The nestin staining pattern in the IZ consisted of bright radial staining of the radial glial processes and fainter staining of axons running parallel to the ventricular surface, as we showed previously (Bott et al., 2019). The sparse 

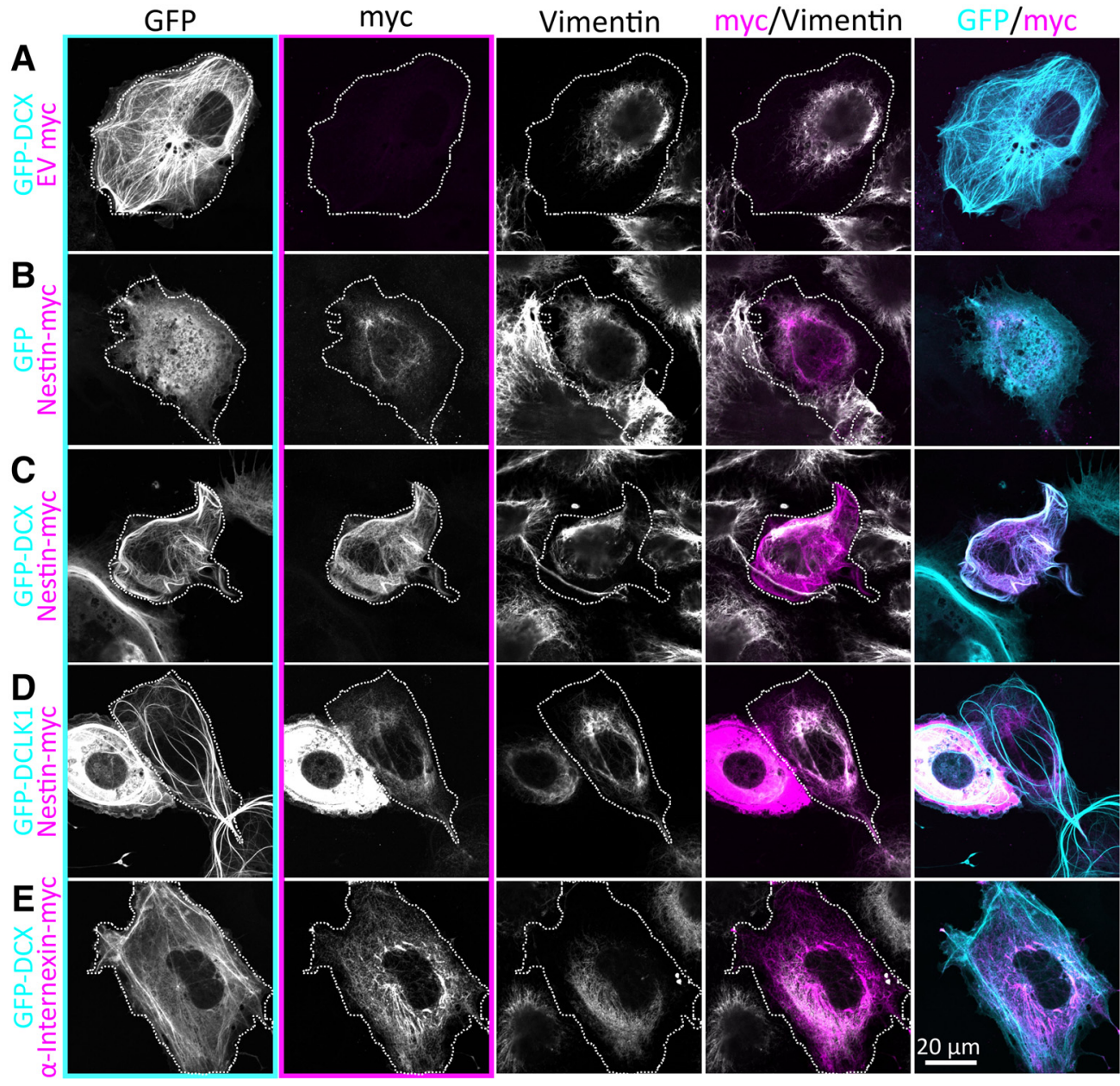

Colocalization

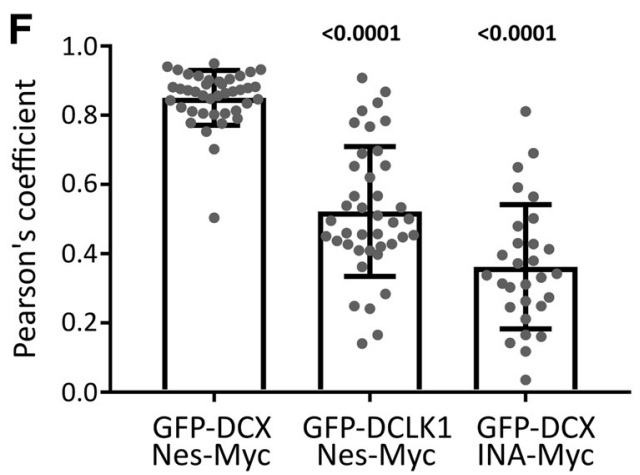

(C)

(D)

(E)

Figure 4. Nestin colocalizes with DCX-MT bundles. $\boldsymbol{A}-\boldsymbol{E}$, Confocal images of Cos7 cells transfected with GFP-DCX + myc EV $(\boldsymbol{A})$, GFP + nes-myc $(\boldsymbol{B})$, GFP-DCX + nes-myc $(\boldsymbol{C})$, GFPDCLK1 + nes-myc $(\boldsymbol{D})$, and GFP-DCX + INA-myc $(\boldsymbol{E})$. All cells are counterstained with GFP booster nanobody, anti-myc tag antibody, and antibody against endogenous vimentin, as labeled above each panel column. The transfected cell is outlined with a dotted line. Individual black and white images are shown for each channel separately, as well as merged images of the GFP/ myc and myc/vimentin channels. Panel outlines of black and white images indicate the color used in the composite images. Nestin is closely colocalized with vimentin in the perinuclear region, except when GFP-DCX is coexpressed, which enriches nestin onto DCX MT bundles. Vimentin remains in the perinuclear region. DCLK1 and INA do not have the same relationship with Nestin or $D C X$, respectively. $\boldsymbol{F}$, Pearson's coefficient of conditions represented in $\boldsymbol{C}-\boldsymbol{E}$ (as indicated below the $x$-axis labels). $N=30-40$ cells from two or three independent experiments are shown with the mean. Error bars indicate SD. Data were analyzed with nonparametric Kruskal-Wallis one-way ANOVA test with Dunn's correction. Each $p$ value shown above each condition was compared with GFP-DCX with Nes-myc (first lane). Both $p$ values are $<0.05$ and are thus in bold. Both nestin with DCLK1, and INA with DCX have significantly less colocalization, as indicated by the smaller Pearson coefficients.

labeling provided by the HA-tag knock-in strategy thus allowed us to identify individual neurons at the earliest stage of axon extension and positioning and to assess for nestin staining. A high magnification of a single section of a $z$ stack shows an example of nestin staining that clearly localized within the distal region of the extending axon (Fig. 5D) but did not extend to the most distal regions of the GC. Orthogonal projections of the $z$ stack were used to allow for visualization of nestin within the 
A
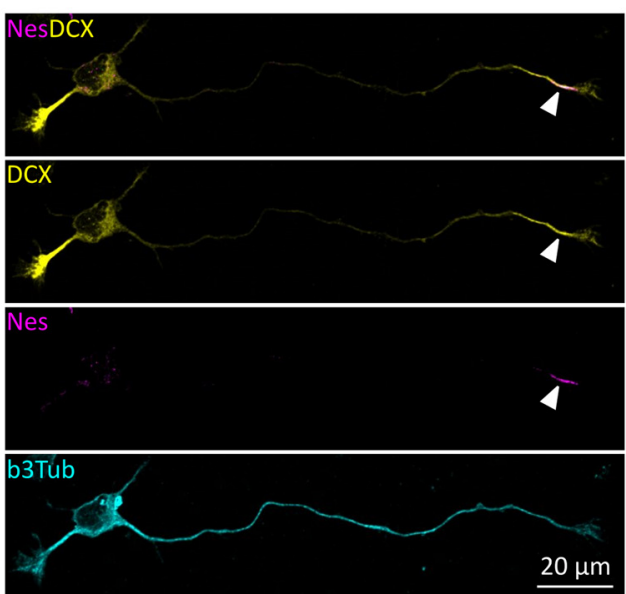

C IUE: E13.5 - fixed at E16.5
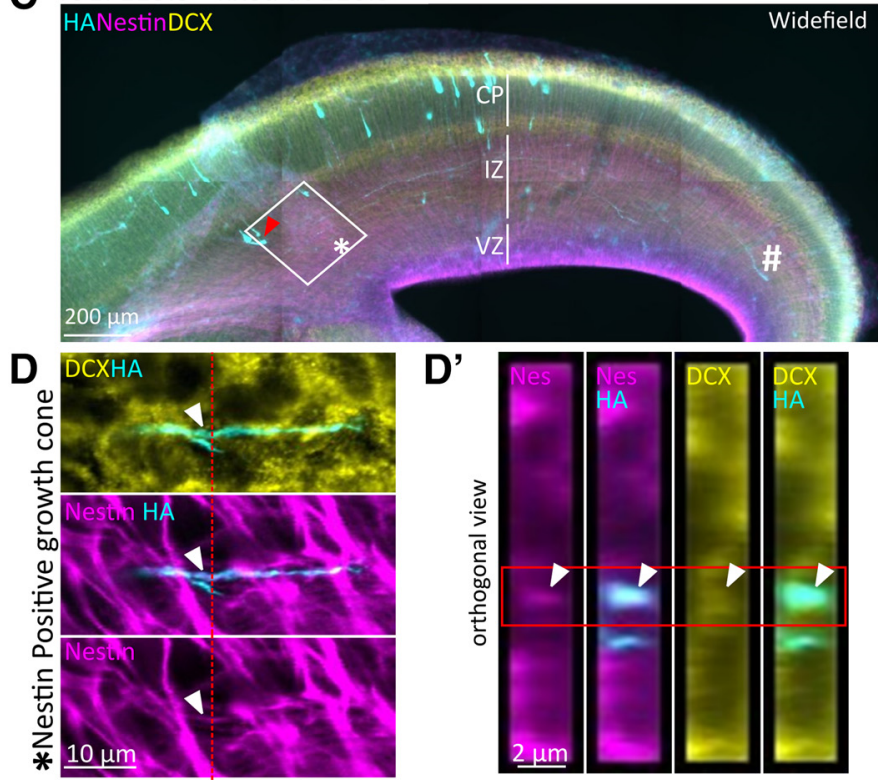

D'

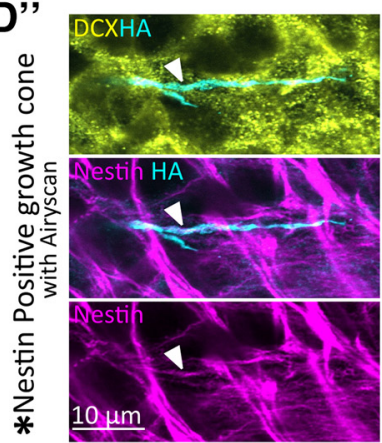

B
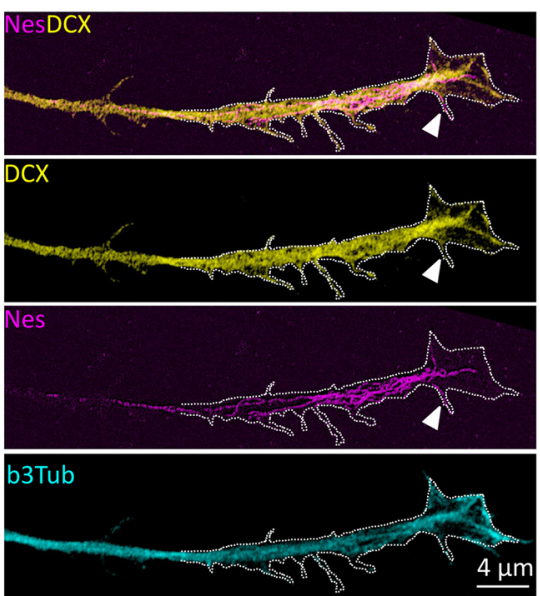

Widefield

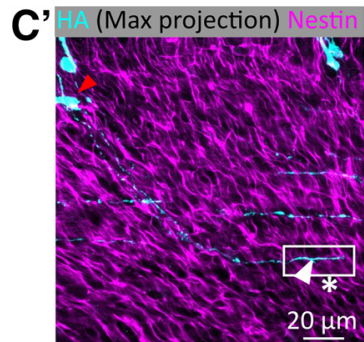

$\mathbf{F}$

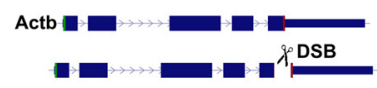

repair by NHEJ

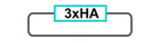

$1=\sin ^{2}$

${ }^{3 \times H A} \lambda^{8}$

ACTB-3XHA

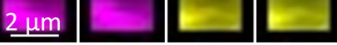

$\mathbf{E}$

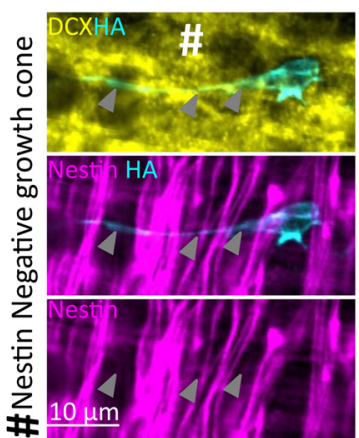

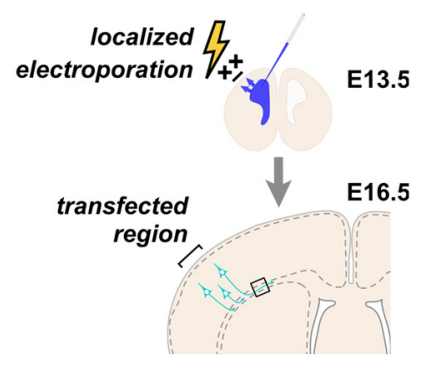

Figure 5. Nestin colocalizes with DCX in axons of newly born neurons in vitro and in vivo. A, Confocal images of an E16 cortical mouse Stage 3 neuron after 1 DIV culture, counterstained for endogenous $D C X$, nestin, and $\beta 3$ tubulin. DCX is enriched at the distal region of all neurites, whereas nestin immunostaining labels only the distal region of the growing axon (arrowhead), but not of the minor neurites. $\boldsymbol{B}$, Enhanced resolution confocal imaging of nestin in the distal region of an axon together with DCX. Distally, some nestin filaments appear to extend into the central region of the $\mathrm{GC}$ and into some proximal filopodia (arrowhead). C, Wide-field immunofluorescence image of a coronal section of E16.5 mouse cortex to illustrate the sparse labeling of individual neurons achieved with knockin of HA-tag using CRISPR and IUE. In all panels, red arrowhead indicates the cell body that projects the HA/Nes/DCX-positive GC. *Nestin-positive GC. "Nestin-negative neuron (shown in $\boldsymbol{D}^{\prime \prime}, \boldsymbol{E}$ ). The major cortical layers are labeled: VZ, Ventricular zone. Antibody labeling is indicated in each panel. $\boldsymbol{C}^{\prime}$, Inset of square in $\mathbf{C}$ demonstrating HA staining of a single Stage 3 neuron with the entirety of its axon imaged in a confocal stack (maximum projection). White arrowhead indicates the distal axon/GC. Red arrowhead indicates the cell body. $\boldsymbol{D}, \boldsymbol{D}^{\prime}$, Confocal images. $\boldsymbol{D}^{\prime \prime}$, Airyscan-enhanced resolution image. $\boldsymbol{D}$, High-magnification zoom of the inset square of $\boldsymbol{C}^{\prime}$ of the GC marked with * in $\boldsymbol{C}$ and $\boldsymbol{C}^{\prime}$. A single $z$ plane is shown. $\boldsymbol{D}^{\prime}$, Orthogonal projection of the region indicated in the red dashed line of $\boldsymbol{D}$. Red box outlines the relevant HA-labeled cell, which is positive for nestin and DCX. $\boldsymbol{D}^{\prime \prime}$, High-magnification Zeiss Airyscan confocal image equivalent of $\boldsymbol{D}$. $\boldsymbol{E}$, High-magnification zoom of the $\mathrm{GC}$ marked with \# in $\boldsymbol{C}$. Arrowheads point along the length of the axon; nestin is not detected in this $\mathrm{GC}$. $F$, Diagram of the experimental strategy to detect nestin and DCX in single GCs in mouse cortex. E13.5 mouse cortex was electroporated in utero to sparsely introduce three HA tags into $\beta$-actin (Actb-3xHA) by CRISPR. Brains were fixed at E16.5. The CRISPR strategy is depicted diagrammatically. 
A DCX phosphorylation by cdk5 requires Nestin and is independent of p35 level
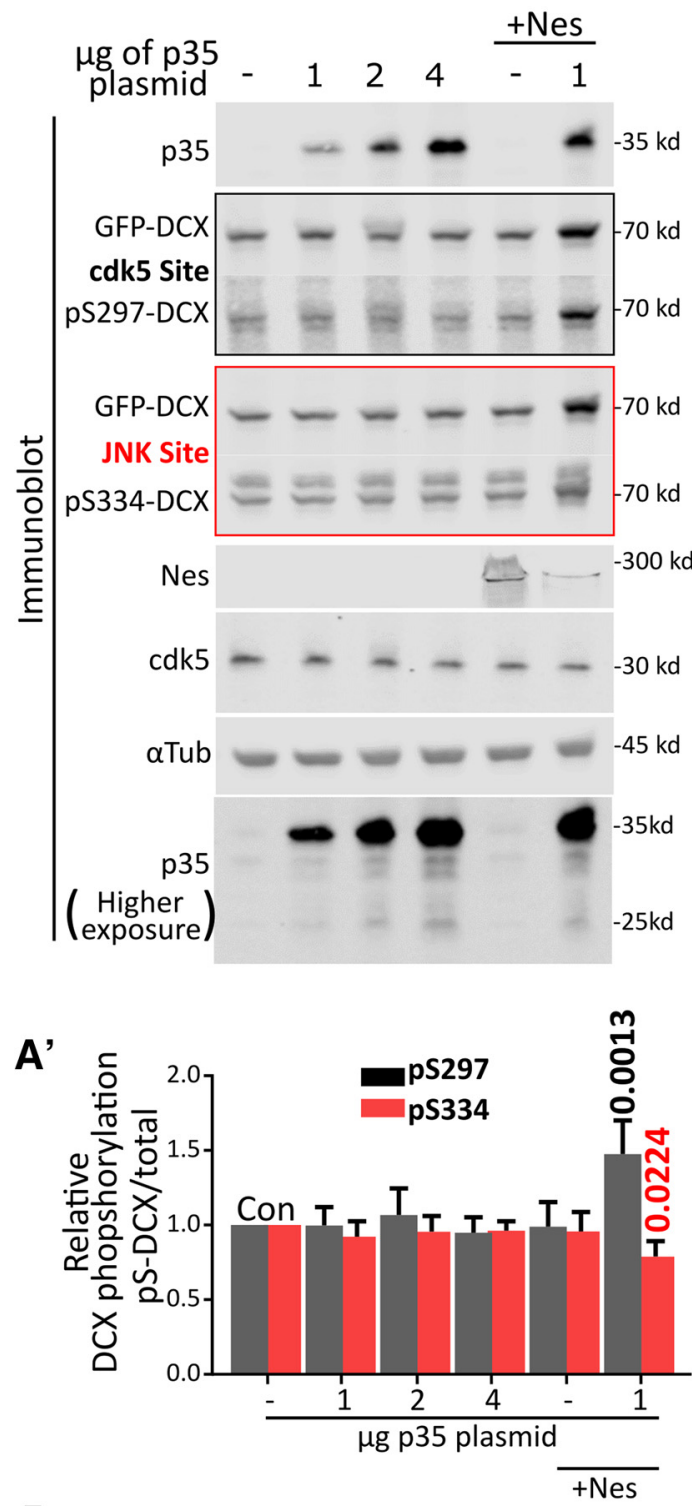

B

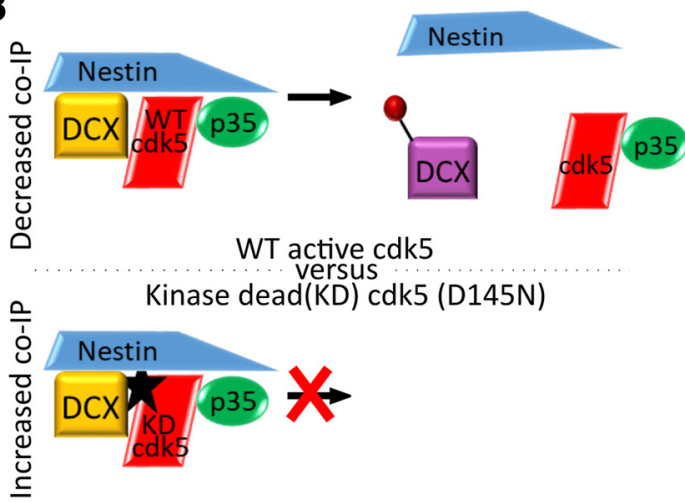

C Cdk5 activity dependent DCX and Nestin interaction

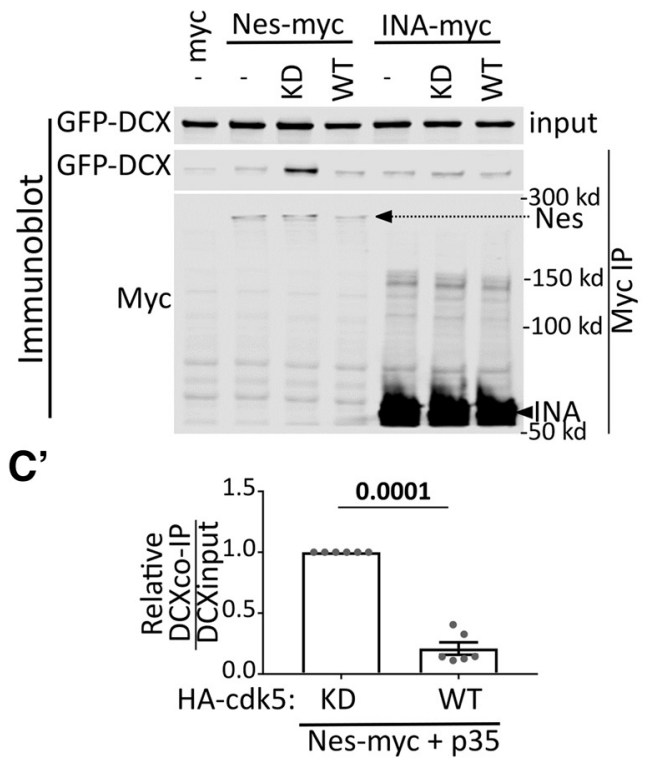

D Nestin increases DCX's interaction with $\mathrm{p} 35$ by co-IP

D'
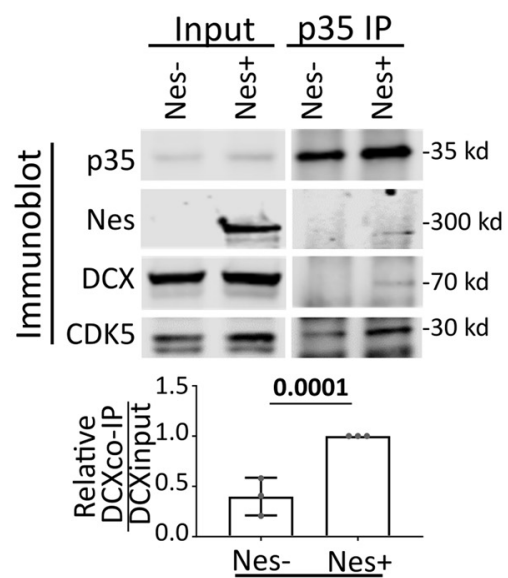

GFP-DCX + p35 +HA-cdk5-KD

$\mathbf{E}$

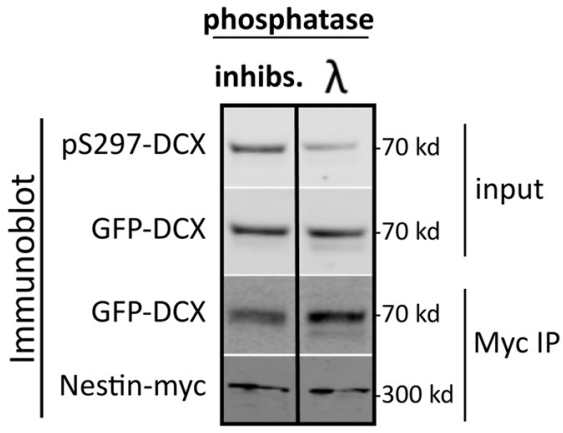

Figure 6. Nestin scaffolds cdk5 and DCX to promote DCX phosphorylation. $\boldsymbol{A}$, Variable amounts of p35 plasmid $(0,1,2,4 \mu \mathrm{g})$ were transfected to increase levels of $\mathrm{p} 35$ expression in the absence of nestin. In addition, nestin was coexpressed with 0 or $1 \mu \mathrm{g}$ p35 plasmid (+Nes lanes). GFP-DCX was expressed in every condition. Levels of pS297-DCX and pS334-DCX were determined by Western blotting. Levels of GFP-DCX, p35, and cdk5 are also assayed. A longer exposure of the p35 blot is shown to illustrate negligible levels of the proteolytic product p25 form of p35. $\boldsymbol{A}^{\prime}$, Densitometry analysis of $\boldsymbol{A}$. Gray bars represent the ratio of pS297-DCX/total GFP-DCX. Red bars represent the ratio of pS334-DCX/total GFP-DCX. Error bars indicate SD. Statistical analysis was performed with one-way ANOVA with Dunnett's correction. Significant pairwise $p$ values compared with control (Con) are indicated in black for pS297 and in red for pS334. All other pairwise comparisons to control were not significant and are not shown. $N=4$ independent experiments. $p$ values of $<0.05$ are indicated in bold. $\boldsymbol{B}$, Experimental model for using the kinase dead (KD) cdk5- D145N to trap substrate: If a quaternary complex of cdk5/p35/DCX/nestin exists, the kinase-dead cdk5 is predicted to trap all components of the complex, which can now be 
HA-positive axon (Fig. 5 $D^{\prime}$ ). Enhanced-resolution Airyscan confocal imaging of the same GC in the same $z$ plane allowed for better appreciation of the axonal nestin (Fig. $5 D^{\prime \prime}$ ). Nestin in distal axons was thus also observable in vivo and thus mirrors what was seen in culture (Bott et al., 2019). DCX protein localization was observed in the same nestin-positive process, but also more broadly in other axons (i.e., throughout different stages of neuron maturation), and is thus seen in more neurons than nestin. We observed nestin primarily in the smaller, more compact GCs of younger neurons whose cell bodies had not yet reached the $\mathrm{CP}$ (Fig. $5 C$, *). The GCs of longer axons (presumably belonging to more mature neurons) did not have detectable nestin enrichment and were larger in size (Fig. 5C,E, \#). Although we were able to observe examples of nestin-positive GCs by this technique, the very low number of definable GCs with traceable axons per electroporated brain made quantification from this preparation not feasible. Therefore, we looked at all the GCs we could identify to see that they were qualitatively comparable with what we had observed in vitro.

\section{Nestin does not increase global cdk5 activity toward DCX}

What is the mechanism for nestin-mediated enhancement of cdk5 phosphorylation of DCX? As was reported before in other systems, we observed that p35 levels were increased by nestin coexpression (Fig. 2), likely due to stabilization of p35 by nestin binding (Sahlgren et al., 2003, 2006; Pallari et al., 2011; Yang et al., 2011). This stabilization of $\mathrm{p} 35$ has been proposed to constitute the mechanism by which nestin promoted cdk5 activity since more activator should result in more cdk5 activity. To test this model directly, we expressed increasing amounts of p35 in HEK293 cells by increasing the amount of p35 plasmid used in the transfection from 1 to $4 \mu \mathrm{g}$. We saw an increased amount of p35 expressed with more p35 plasmid used (Fig. 6A). We could thus directly compare the levels of pS297-DCX at similar p35 levels with or without nestin. Even with similar levels of p35, levels of pS297-DCX were not increased in the absence of nestin (Fig. $6 A, A^{\prime}$, gray bars). This demonstrated that the increased p35 levels were not sufficient to account for the increased DCX phosphorylation by cdk 5 observed with nestin coexpression. Nestin

\section{$\leftarrow$}

coimmunoprecipitated. WT cdk5, on the other hand, more transiently associates with its substrate DCX and does not form a stable complex (as is typical for kinases and their substrates). C, KD cdk5 increases efficiency of nestin pulldowns for DCX. GFP-DCX and p35 were cotransfected with myc EV, nes-myc, or INA-myc together with HA-cdk5-WT or HA-cdk5-KD, as indicated above the lanes, and immunoprecipitated with anti-myc antibody. GFP-DCX inputs (top) were compared with amounts of immunoprecipitated GFP-DCX after anti-myc IPs (bottom two blots). $C^{\prime}$, The amount of DCX after nestin IP is shown as a ratio of the coimmunoprecipitation to input DCX. HA-cdk5-KD was compared with HA-cdk5-WT after nestin IP. This ratio was set to 1 for cdk5-KD. With cdk5-KD, DCX is trapped in a stable complex with nestin. Error bars indicate SD. Statistical analysis was performed with $t$ test. $N=6$ independent experiments. $p$ values of $<0.05$ are indicated in bold. $D$, P35 coimmunoprecipitates more $D C X$ when nestin is coexpressed. Transfection conditions are indicated above each lane. HAcdk5-KD is expressed in each condition to stabilize the kinase/substrate/scaffold complex. Input levels in the lysate (left) are compared with levels of GFP-DCX immunoprecipitated with anti-p35 antibody (right). Nestin increases the amount of DCX that coimmunoprecipitates with $\mathrm{p} 35$. $\boldsymbol{D}^{\prime}$, Relative densitometry analysis of DCX in $\mathrm{p} 35$ coimmunoprecipitation compared with DCX in input. Error bars indicate SD. Statistical analysis was performed with $t$ test with Welch's correction. $N=3$ independent experiments. $E, \lambda$ phosphatase effectively reduces pS297 levels of GFP-DCX as detected in input lysates. Nestin-myc can coimmunoprecipitate more GFP-DCX when dephosphorylated with $\lambda$ phosphatase, compared with untreated samples containing phosphatase inhibitors (inhibs.). Binding was performed in vitro using overexpression lysates. A representative blot (of four independent experiments) is shown. thus did not increase global cdk 5 activity but selectively increased cdk5 activity toward DCX.

\section{Nestin scaffolds cdk5/p35 for DCX phosphorylation}

We next asked how nestin can increase phosphorylation of DCX selectively. We hypothesized that nestin acted to scaffold the active kinase (cdk5/p35) with the substrate by binding to both proteins simultaneously (Fig. $6 \mathrm{~B}$, top, diagram). Typically, interactions between a kinase and its substrate are transient, and phosphorylation of the substrate results in dissociation of the kinase from the substrate (Manning and Cantley, 2002; Goldsmith et al., 2007). Mutated kinases which are "kinase-dead" (KD) form more stable complexes with their substrates, thus "trapping" the substrate bound to the kinase. This will result in an increase in coimmunoprecipitation efficiency of the substrate with the kinase (Fig. $6 B$, bottom). In order to test for a multimeric complex of nestin with cdk5/p35 and DCX, we compared the extent of DCX-nestin complex formation in cells coexpressing either HA-cdk5-WT or HA-cdk5-D145N (KD) with p35 and nestin (Fig. 6C). We could coimmunoprecipitation significantly more DCX with nestin-myc (using anti-myc IP) when the substrate-trapping cdk5-KD was coexpressed, compared with low coimmunoprecipitation efficiency with cdk5-WT (Fig. $6 C^{\prime}$ ). As a control, coexpression of INA-myc, which does not interact with p35 or DCX, resulted in only background levels of GFP-DCX coimmunoprecipitation in all conditions (Fig. 6C). These results suggested that nestin was acting as a scaffold for cdk5 and DCX.

To more directly test this idea, we pulled down the substratetrapped cdk5-KD complex via p35 IP, to test for differences in DCX coimmunoprecipitation in the absence or presence of nestin (Fig. 6D). We found that nestin coexpression significantly increased the amount of DCX recovered in the coimmunoprecipitation by anti-p35 antibody, suggesting a complex of p35cdk5-KD with DCX and nestin (Fig. $6 D, D^{\prime}$ ). Nestin did not affect the abundance of cdk5/p35 heterodimer, as there was not a significant difference in the amount of cdk5 recovered by antip35 IP in the absence or presence of nestin (normalized average of 1 (no nestin) compared with 1.125 (with nestin), $p=0.88$, $n=3)$.

Our model predicts that nestin would bind with higher affinity to dephosphorylated DCX (Fig. 6B, top, diagram). To test this, we treated GFP-DCX containing lysate, prepared from transfected HEK293 cells, with $\lambda$ phosphatase, or with phosphatase inhibitors, to increase dephosphorylated or phosphorylated DCX, respectively (Fig. $6 E$, inputs). These crude lysates were mixed with nestin-myc containing lysates, and nestin-myc was immunoprecipitated. We found that the sample that had been treated with the exogenous $\lambda$ phosphatase (containing more dephosphorylated DCX) was coimmunoprecipitated more efficiently by nestin-myc than the sample that contained a higher level of pS297-DCX (Fig. 6E).

Thus, we propose that nestin acts as a selective scaffold, which can bind to cdk5/p35 and DCX simultaneously, and this results in increased efficiency of WT cdk5-mediated phosphorylation of DCX (see Fig. 11). Dephosphorylation of DCX was shown by others (Shmueli et al., 2006; Bielas et al., 2007) to be dependent on the recruitment of PP1 to DCX by the actin binding protein SPN. We confirmed this observation (Fig. $2 A, A^{\prime}$ ). Thus, we propose that the level of DCX phosphorylation is determined by the balance between these opposing reactions (see Fig. 11, top) and that phosphorylation would be more dominant when nestin is present. We thus envision nestin to act like a "gain control" in GCs, which sensitizes DCX to cdk5 phosphorylation. 
The T316D mutation in nestin affects p35 and DCX binding as well as DCX phosphorylation, but not incorporation into vimentin filaments

We previously showed that nestin expression is associated with small compact GCs, whereas nestin negative-neurons have larger GCs (Bott et al., 2019). Is the effect of nestin on GC morphology dependent on its ability to scaffold cdk5/p35/DCX? To test this, we aimed to create nestin mutants that lack cdk5/p35 binding. Previous work showed that the threonine at position 316 (T316) on nestin is an important regulatory site for $\mathrm{cdk} 5 / \mathrm{p} 35$ binding and for cdk5-dependent effects of nestin (Yang et al., 2011; Su et al., 2013). The T316 site can be phosphorylated, which affects binding to $\mathrm{p} 35$. The T316A phospho-dead mutant had increased binding to $\mathrm{p} 35$ in in vitro binding assays of purified recombinant protein (Yang et al., 2011). In addition to making the T316A phospho-dead nestin mutant, we created a phospho-mimetic mutant (T316D-Nes), reasoning that it might be unable to bind p35. To test p35 binding of the T316 mutants, we used a coimmunoprecipitation strategy similar to that used in Figure 1 (Fig. $7 A$ ). In our hands, the T316A-Nes mutant did not show a consistent increase in p35 binding. T316D-Nes, on the other hand, was greatly deficient in p35 binding (Fig. $7 A, A^{\prime}$ ). We then tested whether these nestin mutant proteins still formed a complex with DCX. We performed IPs with WT nestin or nestin mutants when GFP-DCX was coexpressed. Surprisingly, T316D-Nes exhibited decreased binding to DCX (Fig. $7 A, A^{\prime}$ ), whereas T316A-Nes still coimmunoprecipitated DCX. We note that T316D-Nes levels appeared consistently lower in the lysates compared with WT nestin or T316A-Nes.

In order to test the ability of T316 nestin mutants to associate with DCX by an additional assay, we also used the Cos7 cell immunofluorescence assay to determine whether nestin mutants were still recruited to MTs by DCX. In agreement with the coimmunoprecipitation studies, both WT nestin and T316A-Nes did not have significantly different colocalization means with GFPDCX MT bundles, when quantified with Pearson's coefficient (Fig. 7H). T316D-Nes, on the other hand, localized more similarly to vimentin and did not appear enriched with DCX-decorated MTs (Fig. 7B-D). T316D-Nes had significantly $(p=0.0001)$ lower Pearson's coefficient mean compared with WT nestin (Fig. $7 H$ ).

Our scaffolding hypothesis proposes physical associations between DCX, p35/cdk5, and nestin. We thus reasoned that a nestin mutant deficient in p35 and DCX binding should also be deficient in promoting cdk5-mediated phosphorylation of DCX. We tested whether the pS297-DCX levels were altered in cells expressing WT or T316 nestin mutants (Fig. 7I). T316A-Nes + p35 resulted in increased phosphorylation of DCX similar to WT nestin + p35, whereas T316D-Nes + p35 did not result in increased phosphorylation of DCX (Fig. 7I, quantified in Fig. $\left.7 I^{\prime}\right)$. This result is consistent with our scaffolding hypothesis.

Since nestin forms heteropolymers with vimentin, we also tested whether the nestin T316 mutants still interacted with vimentin. The T316 site is just outside of the IF polymerization rod domain, so modification of this site is not expected to interfere with IF polymerization. Indeed, we observed WT nestin, T316A-Nes, and T316D-Nes coimmunoprecipitating vimentin similarly (Fig. $7 A, A^{\prime}$ ), arguing that they still formed homooligomers/polymers with vimentin. In agreement, these mutants appeared to codistribute with endogenous vimentin filaments similarly to WT nestin when coexpressed in Cos7 cells (Fig. 7E$G)$. We thus conclude that T316D-Nes binds less well to both p35 and DCX, whereas T316A-Nes still binds p35 and DCX, and that neither mutation grossly disrupts vimentin binding. We used the T316D-Nes mutant to test the role of the nestin-p35DCX complex in neurons below.

\section{Binding to $\mathrm{p} 35$ and/or DCX is required for nestin's effect on GC size}

We previously reported that reducing neuronal nestin levels leads to larger GCs (Bott et al., 2019). We thus wondered whether overexpression of WT nestin would do the opposite (i.e., lead to smaller GCs). We expressed myc-nestin in cultured cortical neurons for 2 days along with GFP to allow for detection of transfected cells. At this stage, the majority of neurons are nestin-negative (Bott et al., 2019), allowing us to assess the effects of reexpressing nestin. Overexpression of WT nestin-myc resulted in statistically smaller GCs on axons (arrows) with fewer filopodia, which is the opposite phenotype of downregulating nestin. Overexpression of INA-myc did not change GC size or filopodia number compared with myc-EV controls (Fig. 8A,B, $B^{\prime}$ ). Next, we asked whether p35/DCX binding by nestin was required for the effect on GC size. T316A-Nes, which retained binding to p35 and DCX (Fig. 7A) and resulted in WT levels of DCX phosphorylation (Fig. $7 B$ ), resulted in small GCs with fewer filopodia, similar to WT nestin (Fig. $8 A, B, B^{\prime}$ ). However, the T316D-Nes mutant, which did not interact with p35 or DCX (Fig. $7 A$ ) and did not result in increased DCX phosphorylation (Fig. $7 B$ ), did not significantly alter GC size or filopodia number (Fig. $8 A, B$, $\left.B^{\prime}\right)$. Therefore, this result suggests that binding to p35 and/or DCX is required for nestin's effect on GC size.

\section{Nestin does not have any effect on GC size in the absence of its downstream effector DCX}

Since nestin selectively bound to DCX and affected its phosphorylation, we hypothesized that the effects of nestin overexpression on GCs were dependent on DCX. Therefore, we tested whether nestin overexpression would affect GCs in the absence of DCX. We thus overexpressed nestin-myc, myc-EV, or myc-INA in WT or Dcx KO neurons and determined GC size and filopodia number. We again observed decreased axonal GC size (arrows) and decreased filopodia number specifically with nestin-myc expression (Fig. 9A, $A, B^{\prime}$ ). However, in $D c x$-null neurons, no decrease in GC size or filopodia number was seen with nestin-myc expression (Fig. 9A,B,B'). When GFP-DCX was reintroduced together with nestin-myc into $D c x$-null neurons, the nestin effect on GC size and GC filopodia was rescued (Fig. 9A,B, $B^{\prime}$ ). Axon length was not affected (Fig. $9 B^{\prime \prime}$ ). We thus conclude that nestin acts via DCX to influence GC size.

\section{DCX is a cdk5 substrate downstream of Sema3a, and nestin sensitivity to Sema3a is absent in $D c x$-null neurons}

We previously showed that 1 DIV cortical WT neurons respond to Sema3a by retracting their axon GC filopodia and that this response is blunted when nestin is downregulated. We wondered whether DCX was also downstream of nestin for Sema3a responsiveness. S297-DCX phosphorylation has been reported to be induced by Sema3a in DRG neurons (Ng et al., 2013), but this has not been shown in other neuronal cell types. We treated DIV1 E16 cortical mouse neurons with vehicle or $1 \mathrm{~nm}$ Sema3A for 5,15 , or $30 \mathrm{~min}$ and determined the levels of DCX phosphorylation. We could detect phosphorylation of the S297-DCX epitope in cortical neurons, which was significantly increased after Sema3a treatment (Fig. 10A, $A^{\prime}$, gray bars). The JNK site pS334 was not increased after Sema3a treatment (Fig. 10A, $A^{\prime}$, red bars). 

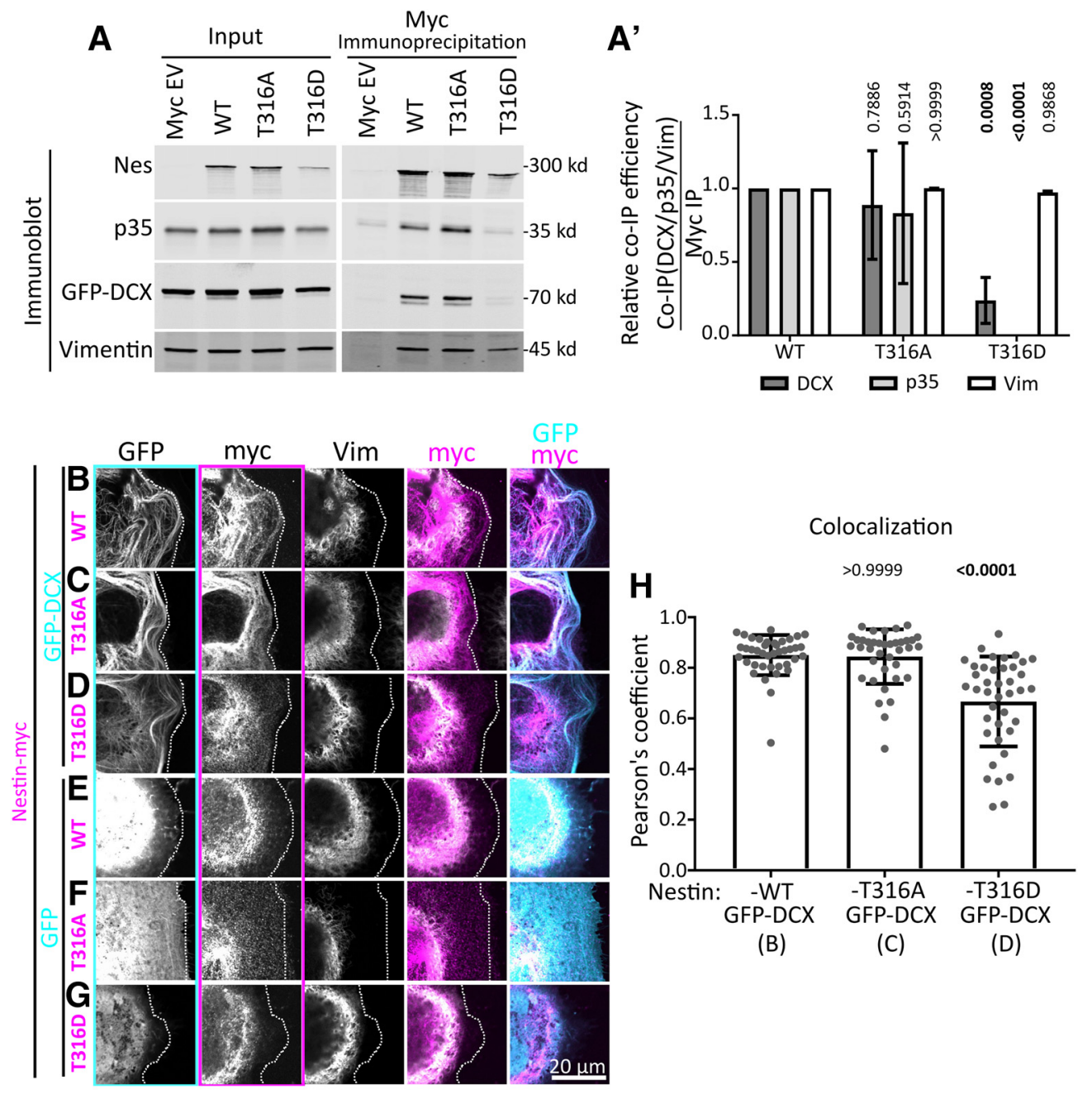

(B)

(C)

(D)
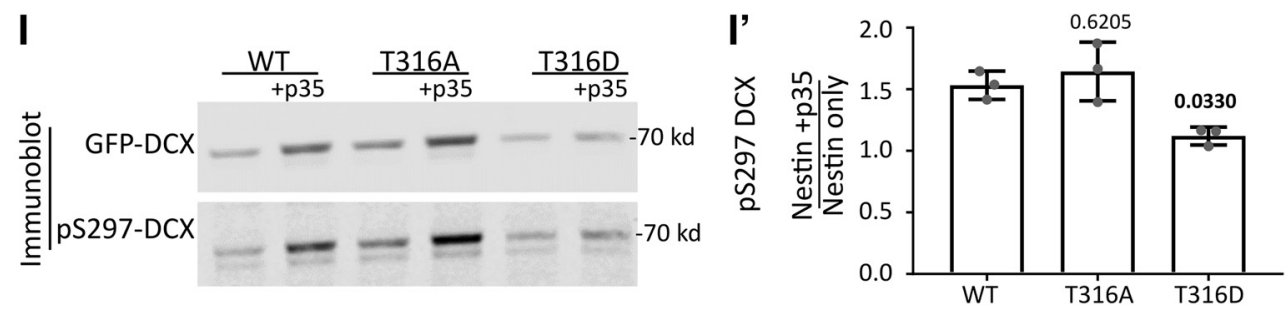

Figure 7. The T316D mutation in nestin affects p35/DCX binding and DCX phosphorylation, but not incorporation into vimentin filaments. $\boldsymbol{A}$, Point mutants in nestin (T316A, phospho-dead; or T316D, putative phospho-mimetic) were tested for binding to $\mathrm{p35}, \mathrm{DCX}$, and vimentin by coimmunoprecipitation from HEK293 cells. Inputs (left) and corresponding coimmunoprecipitation of vimentin, GFP-DCX, and p35 after anti-myc IP (right). The p35 and DCX/vimentin panels are combined from the same experiment, conducted from two different plates of cells. The blots are qualitatively similar for both sets, and were combined into a single figure for simpler viewing. $\boldsymbol{A}^{\prime}$, Densitometry quantification of coimmunoprecipitation efficiency (coimmunoprecipitation/IP) from the blots in $A$. T316D-nestin pulls down greatly reduced levels of p35 and DCX compared with WT nestin. Coimmunoprecipitation of p35 and DCX by T316A-nestin is not significantly different compared with WT nestin. Each mutant coimmunoprecipitates vimentin similarly to WT. Means of $n=3$ experiments for each IP condition. Error bars indicate SD. Data were analyzed with parametric two-way ANOVA test with Tukey's correction. $p$ values shown above each condition compared with the corresponding WT-Nestin coimmunoprecipitation. $p$ values $<0.05$ are in bold and are considered significant. $\boldsymbol{B}-\boldsymbol{G}$, Confocal images of Cos7 cells transfected with GFP-DCX $(\boldsymbol{C}-\boldsymbol{E})$ or GFP $(\boldsymbol{F}-\boldsymbol{H})$ cotransfected with WT nes-myc $(\boldsymbol{C}, \boldsymbol{F})$ or mutant nestin (T316A: $\boldsymbol{D}, \boldsymbol{G}$; T316D: $E, \boldsymbol{H})$, as indicated. The transfected cell is outlined with a dotted line. Individual black and white channels are shown, as well as merges with GFP/myc and myc/vimentin channels. Outlines of black and white images indicate the color used in the composite images. WT nestin and the 316A-nestin mutant colocalize with DCX MT bundles. However, nestin is associated with vimentin when expressed without DCX or when the DCX-binding-deficient T316D-nestin is expressed, even in the presence of DCX. $\boldsymbol{H}$, Pearson's coefficient of conditions represented in $\boldsymbol{B}$ - $\boldsymbol{D}$ (as indicated below the $x$-axis labels). $N=38-40$ cells from three independent experiments are shown with the mean. Error bars indicate SD. The WT nestin with DCX condition has the same values from Figure 4F. Data were analyzed with nonparametric Kruskal-Wallis one-way ANOVA test with Dunn's correction, with each $p$ value shown above each condition compared with GFP-DCX with WT Nes-myc (first lane). $p$ values $<0.05$ and are in bold and are considered significant. The Pearson coefficient mean for Nes-T316A with DCX is not significantly different that Nes-WT with DCX. However, Nes-T316D with DCX has a significantly $(p=0.0001)$ lower Pearson coefficient mean than Nes-WT with DCX. I, Nestin-dependent cdk5 phosphorylation of DCX in HEK293 cells. $\boldsymbol{I}^{\prime}$, Densitometry quantification of the blot in $\boldsymbol{B}$ showing the relative levels of pS297-DCX phosphorylation with expression of each mutant. Each value represents the relative phosphorylation of DCX when expressed with nestin and p35, compared with just nestin expression. T316D-nestin does not augment p35/CDK5-mediated DCX phosphorylation. Error bars indicate SD. Each condition was compared with WT nestin condition (one-way ANOVA with Dunnett's correction). $N=3$ independent experiments. Bold $p$ values are $<0.05$ and are considered significant. 


\section{B Growth Cone Area}
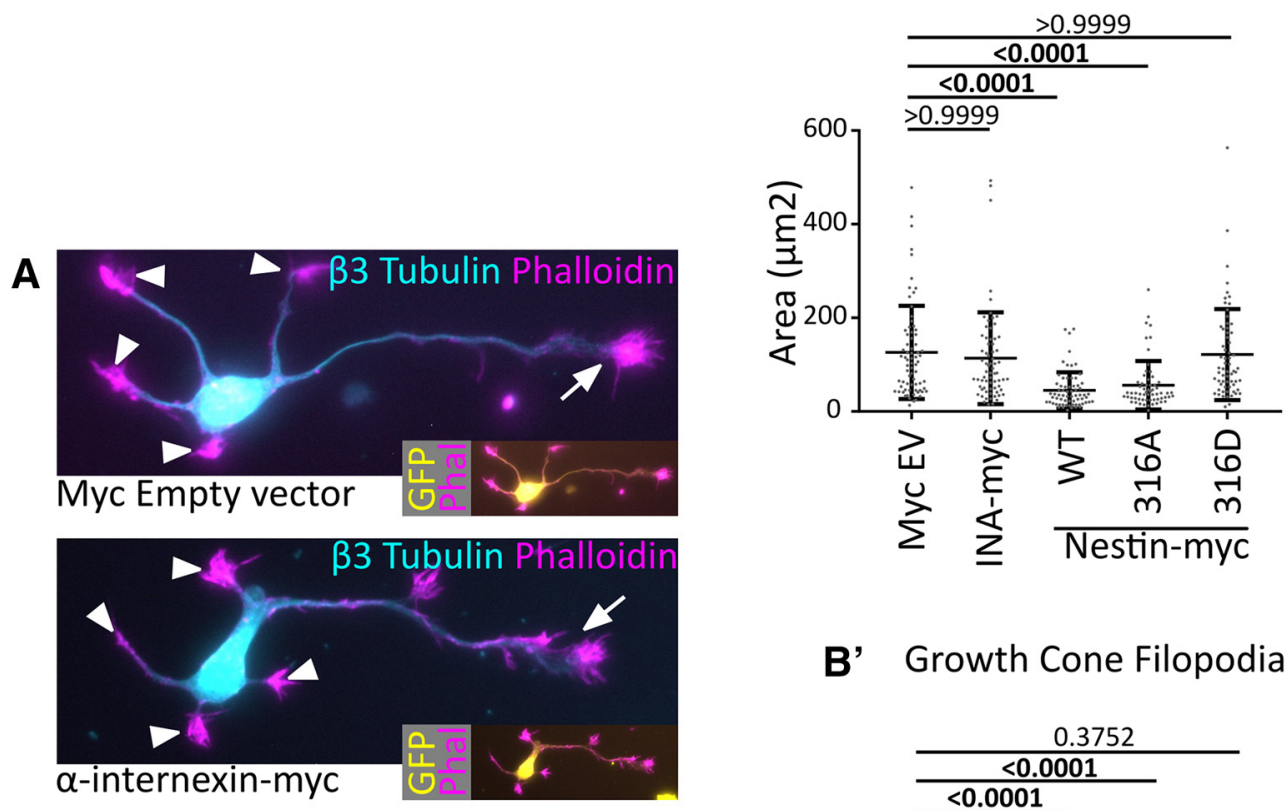

\section{B' Growth Cone Filopodia}

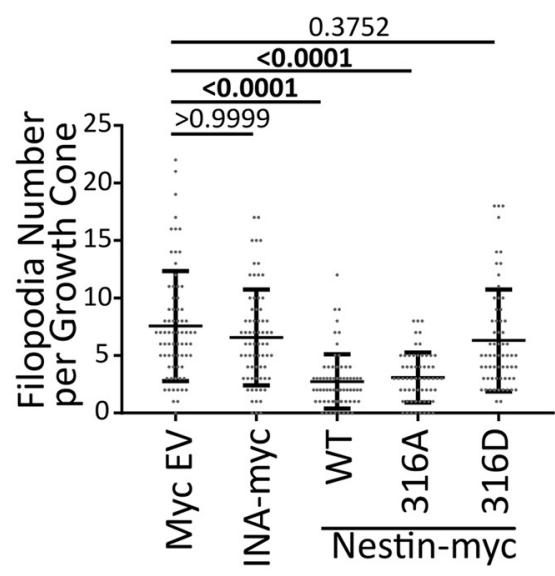

B' Axon Length
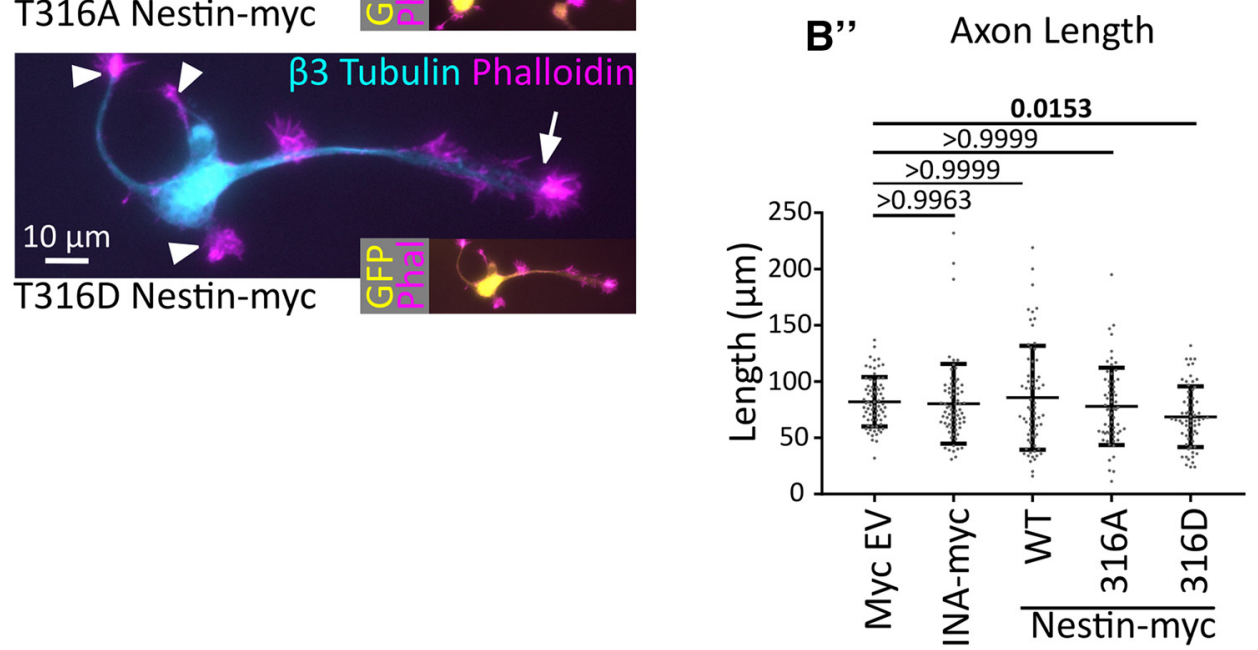

Figure 8. Binding to $\mathrm{p} 35$ and/or DCX is required for nestin's effect on GC size. A, Representative images of DIV2 cortical neurons expressing nestin or T316-nestin mutants compared with EV control or INA-myc. $\beta 3$ tubulin and phalloidin were used to visualize MTs and F-actin, respectively. Arrows indicate axonal GCs. Arrowheads indicate secondary neurites (dendrites). Brightness was adjusted for each image for easier viewing. Insets, Transfected neurons, identified by GFP expression. $\boldsymbol{B}, \boldsymbol{B}^{\prime}, \boldsymbol{B}^{\prime \prime}$, Quantification of neuronal morphology in neurons expressing different nestin constructs (as shown in $A$ ). $N=72-61$ cells counted per condition in three different independent experiments. Data were analyzed with nonparametric Kruskal-Wallis one-way ANOVA test with Dunn's correction. Pairwise $p$ values are shown for comparisons to myc EV (control). WT nestin and T316A-nestin decrease GC area and filopodia number, but T316D-nestin does not. $p$ values of $<0.05$ are indicated in bold. 
A
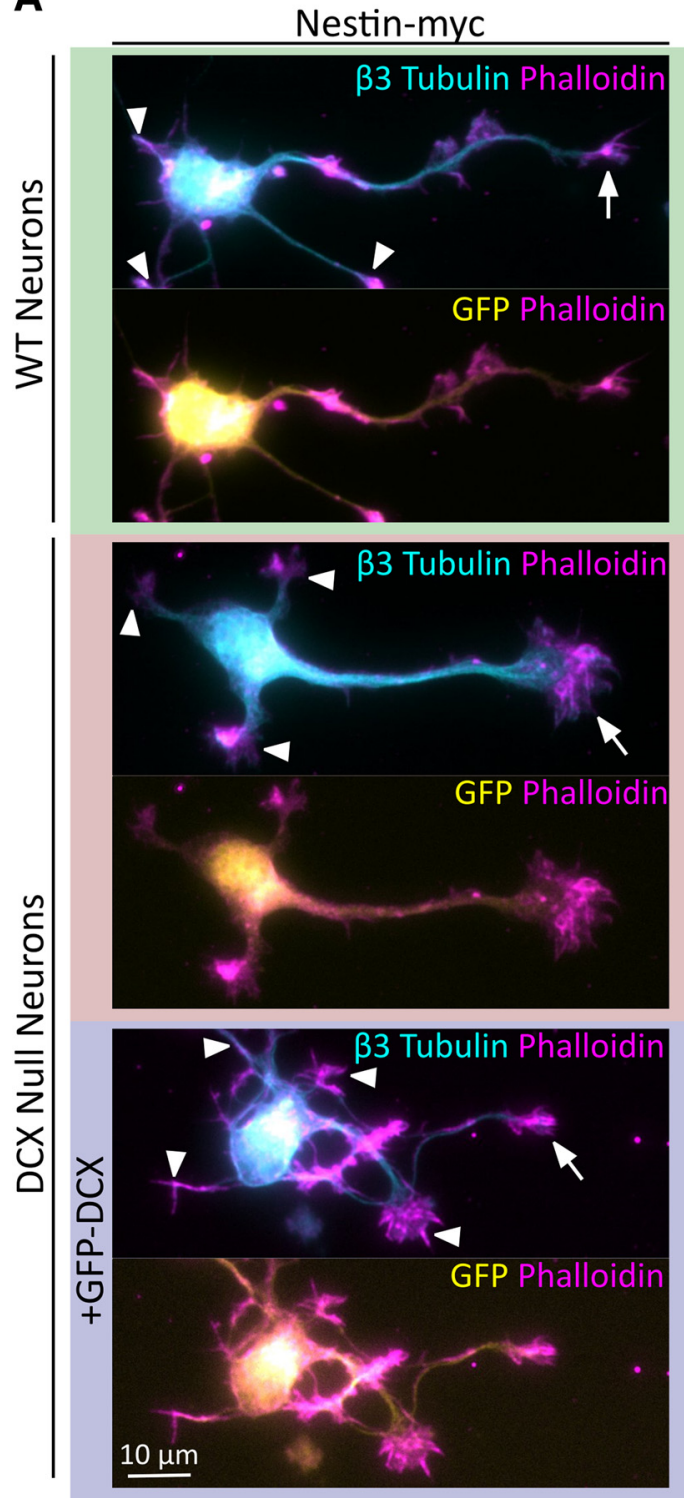

B

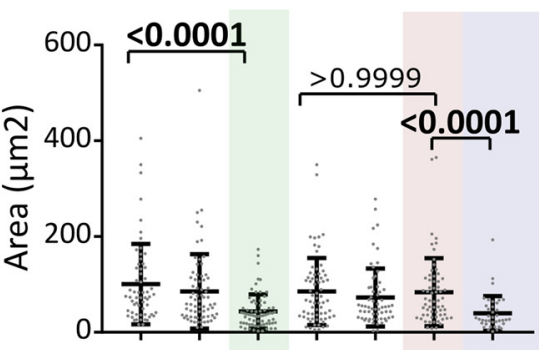

B'

Growth Cone Filopodia

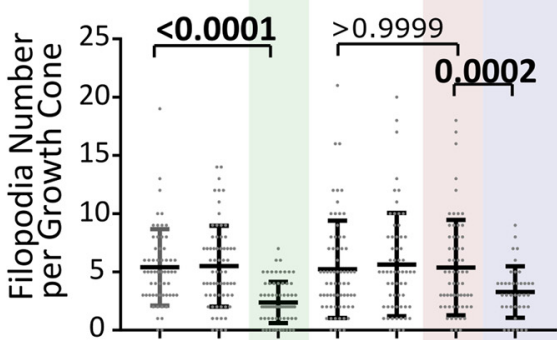

B'"

Axon Length

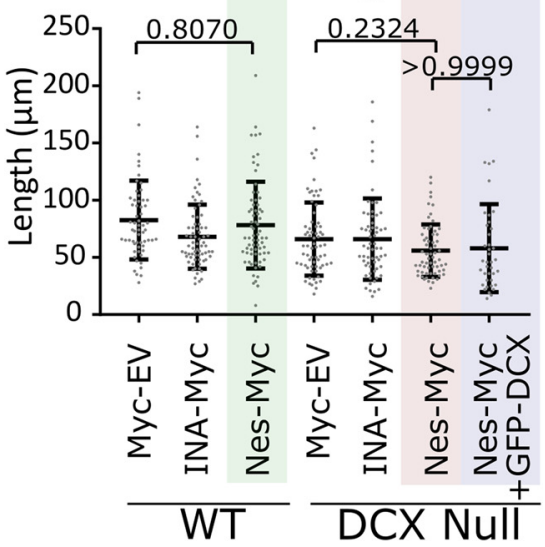

Figure 9. In the absence of $D C X$, nestin overexpression does not result in small GCS. A, Representative images of WT or DCX-null E16 cortical neurons (as labeled) expressing nes-myc with GFP or nes-myc with GFP-DCX after $48 \mathrm{~h}$ in culture. WT neurons transfected with nes-myc show smaller axonal GCs (arrows), whereas Dcx K0 neurons transfected with nestin-myc do not show smaller GCS. When GFP-DCX is cotransfected into Dcx K0 neurons, GCs are smaller. Arrows indicate axon GCs. The GCs of secondary neurites (dendrites) were not considered and are indicated with arrowheads. $\boldsymbol{B}^{\prime}, \boldsymbol{B}^{\prime \prime}, \boldsymbol{B}^{\prime \prime \prime}$, Quantification of morphologic characteristics. The background shading color behind the images in $\boldsymbol{A}$ represent the conditions shaded in $\boldsymbol{B}^{\prime}, \boldsymbol{B}^{\prime \prime}$, and $\boldsymbol{B}^{\prime \prime \prime}$ for easier interpretation. $\boldsymbol{B}^{\prime}, \boldsymbol{B}^{\prime \prime}, \boldsymbol{B}^{\prime \prime \prime}$, Quantification of morphologic measurements from transfected neurons: $\mathrm{GC}$ area $\left(\boldsymbol{B}^{\prime}\right)$, filopodia number $\left(\boldsymbol{B}^{\prime \prime}\right)$, and axon length $\left(\boldsymbol{B}^{\prime \prime \prime}\right)$. Error bars indicate SD. Kruskal-Wallis one-way ANOVA nonparametric test was used. $p$ values are only shown for selected statistical comparisons to keep the graph less cluttered. $N=40-70$ cells counted in three different independent experiments.

pS297DCX, but not pS334DCX levels, were reduced by roscovitine, consistent with activation of cdk5 downstream of Sema3a (Fig. 10A, $A^{\prime \prime}$ ).

In order to determine whether nestin still affected the extent of Sema3a sensitivity in the absence of DCX, we compared the Sema3a response of nestin-positive and nestin-negative neurons in WT or Dcx-null cortical cultures. At 1 DIV, a little more than half of the Stage 3 neurons are nestin-positive, whereas the remainder are nestin-negative (Bott et al., 2019), allowing us to compare Sema3a responsiveness in the same culture when stained against nestin. In WT neurons, we again saw a nestin-dependent decrease in GC size in untreated cultures, whereas the GC size of nestin-positive and nestin-negative neurons was not significantly different in $D c x$-null cultures (Fig. 10B, quantified in Fig. 10C), in agreement with the data in Figure 9. As we reported previously (Bott et al., 2019), we again observed a decrease in filopodia number in nestin-expressing neurons when treated with Sema3a, whereas nestin-negative neurons were less Sema3a-responsive (Fig. $10 C^{\prime \prime}$ ). When we treated $D c x$-null neurons with Sema3a, however, we no longer observed decreased GC filopodia number in nestin-positive neurons. Nestin-positive and nestin-negative neurons derived from $D c x$-null mice showed the same Sema3a sensitivity (in Fig. $10 C^{\prime \prime}$ ), indicating that DCX is required for nestin-dependent regulation of GC size and Sema3a sensitivity. Axon length was not significantly different (Fig. 10C'). 
A 1DIV cortical mouse neurons 1nM Sema3a bath treatment over time ( $\mathrm{min}$ )

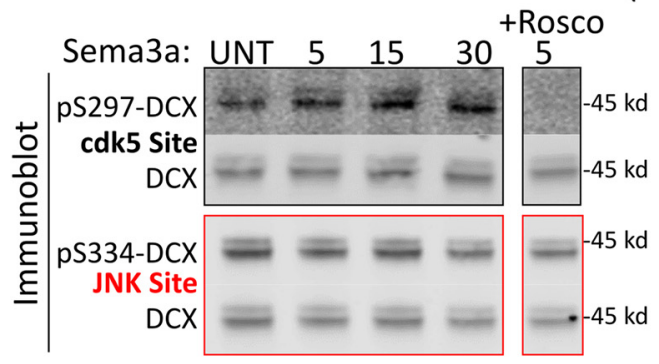

B
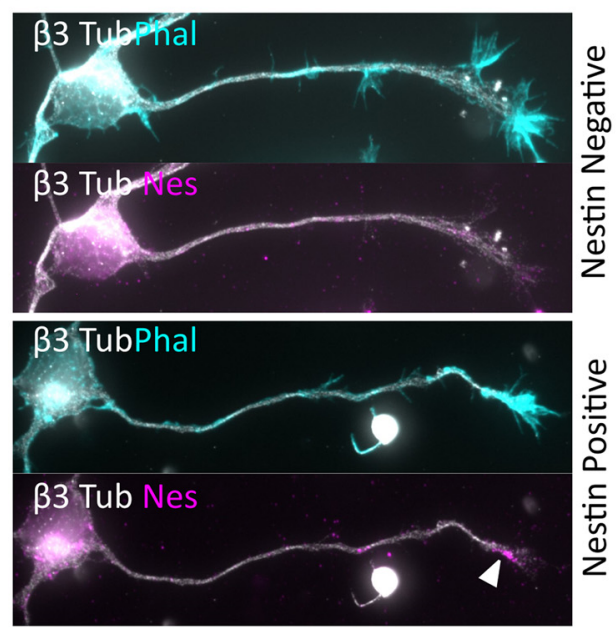

DCX Null Neurons
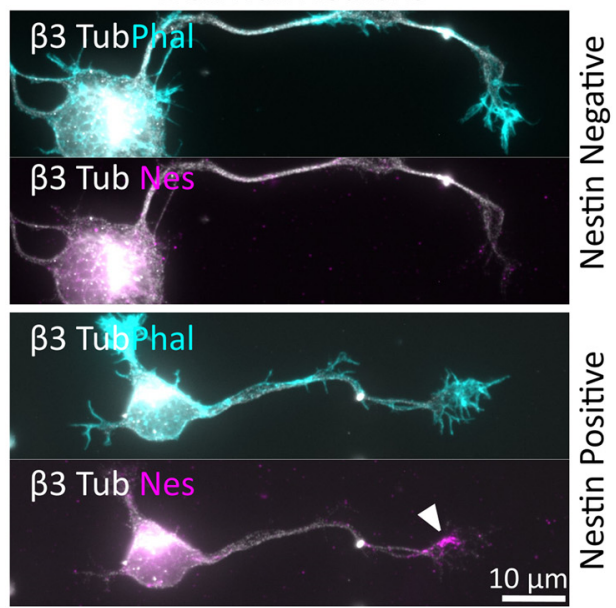

$\mathbf{A}^{\prime}$

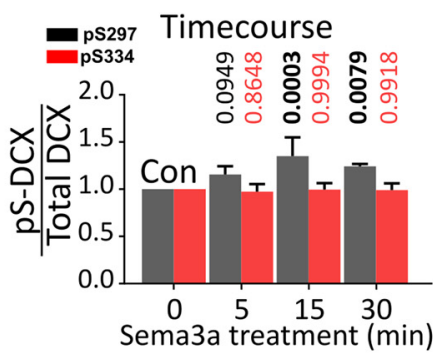

A'”

Roscovitine

(cdk5 inhibitor)

Treatment

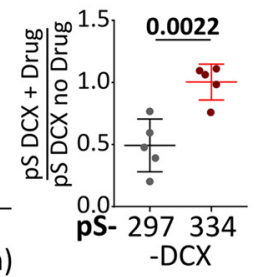

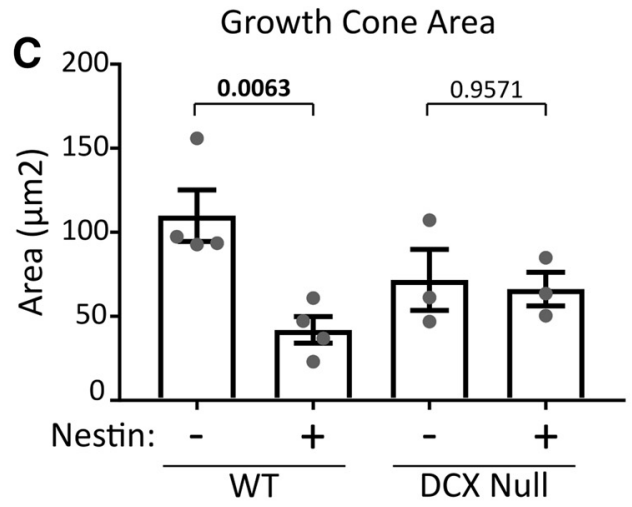

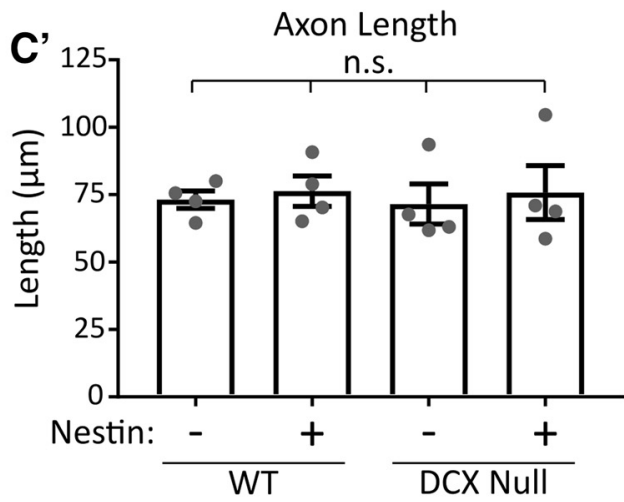

Growth Cone Filopodia

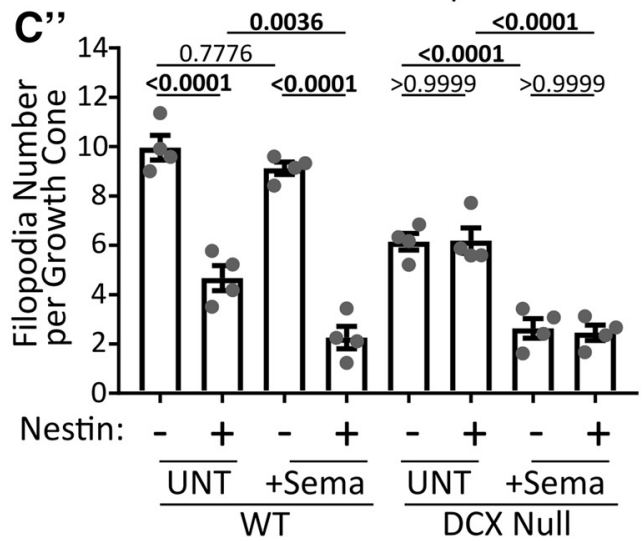

Figure 10. DCX is a cdk5 substrate downstream of Sema3a, and Dcx-null neurons respond in a nestin-independent manner. $\boldsymbol{A}$, Sema3a treatment of 1 DIV cultured mouse cortical E16 neurons significantly increases DCX phosphorylation at S297, but not at S334. Rightmost lane, Neurons were treated with 1 nm Sema3a for 5 min with preincubation of the cells with $10 \mu \mathrm{m}$ roscovitine for 30 min. The inhibitor lane was from the same blot as the other lanes, but separated by intervening lanes that were removed. $\boldsymbol{A}^{\prime}, \mathrm{Quantification}$ of Sema3a time course. $\boldsymbol{A}$, Blots were subjected to densitometry analysis, and the ratio of phospho-DCX signal relative to the total DCX is plotted. DCX phosphorylation at pS297, but not at pS334, begins quickly after Sema3a stimulation and becomes significant at 15 and 30 min. Error bars indicate SD. Statistical analysis was performed at each time point compared with untreated (UNT) with an ordinary one-way ANOVA with Dunnett's correction. $n=5$ independent experiments. $p$ values of $<0.05$ are indicated in bold. $\boldsymbol{A}^{\prime \prime}$, Quantification of roscovitine sensitivity (A, rightmost lane). Each value was obtained 
In our model (Fig. 11), we propose that basally, non-phospho-DCX binding to MTs stabilizes the MTs; and in the absence of nestin-scaffolded cdk5 phosphorylation, DCX provides resistance or buffering against Sema3a-mediated GC collapse and filopodial retraction. Activation of the pathway in nestin-positive WT neurons allows for DCX phosphorylation and removal of the MT-stabilizing effects of DCX. When DCX is absent and is not there to provide resistance to Sema3a, GCs are responsive to Sema3a in a non-nestin-dependent manner.

\section{Discussion}

A number of IF subtypes are expressed in the brain, but their cell biological functions during development are incompletely understood (Yuan and Rao, 2012; Bott and Winckler, 2020). One of the earliest expressed IFs in the developing brain is nestin, but little is known about nestin's function, even in NPCs where it is highly expressed (Park et al., 2010; Mohseni et al., 2011; Wilhelmsson et al., 2019). We previously found that nestin protein expression persists at low levels in early neurons and that nestin expression influences GC size and responsiveness to Sema3a in a cdk5-dependent manner (Bott et al., 2019), but the molecular pathways for this phenotype were not known. Nestin is known to directly interact with the $\mathrm{cdk} 5 / \mathrm{p} 35$ heterodimer. We thus hypothesized that nestin might also affect the activity of cdk5 in neurons, similarly to what occurs during neuromuscular junction development (Yang et al., 2011). Hence, we screened known neuronal $c \mathrm{dk} 5$ substrate cytoskeletal proteins for nestin binding and identified DCX as a novel nestin-associated protein that is preferentially phosphorylated in the presence of nestin and cdk5/p35. Mechanistically, we find that nestin selectively promotes robust phosphorylation of DCX by cdk5/p35 through scaffolding. We show that DCX and nestin colocalize in the distal axon and GC, and that DCX is required for the effects of nestin on GC morphology and Sema3a sensitivity. In summary, our results uncover a novel signaling role for neuronal nestin in regulating GC morphology specifically via scaffolding the cdk5 substrate DCX.
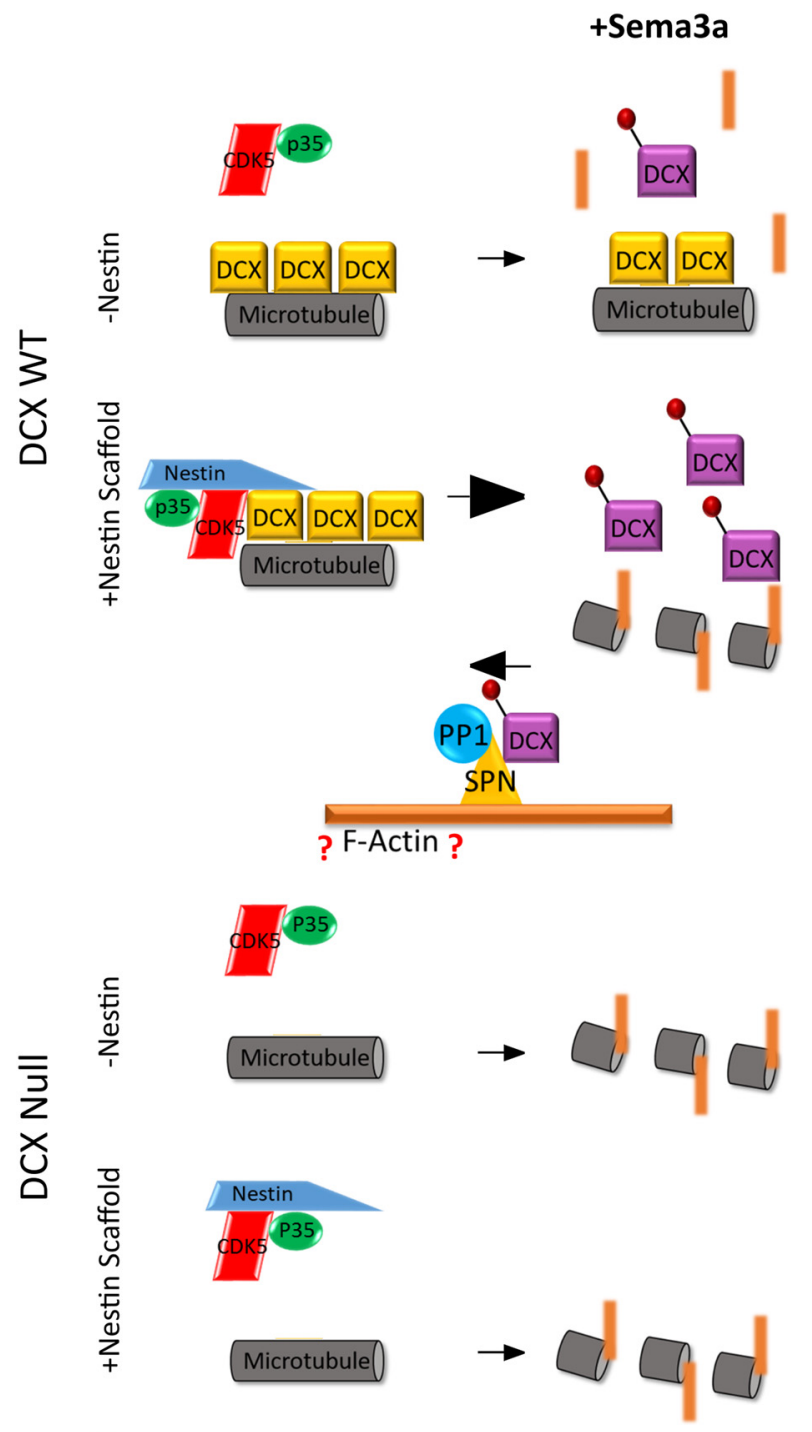

non-nestin dependent MT de-stabilizing factors

from densitometry analysis of Western blots, where the relative level of $p-D C X$ was compared with total DCX. The values in the plot are a ratio of the relative phosphorylation after inhibitor (drug) treatment compared with no inhibitor treatment. Error bars indicate SD. Statistical analysis was performed with a two-tailed unpaired $t$ test. $N=5$ independent experiments. $\boldsymbol{B}$, Representative images of nestin-positive or -negative individual neurons from WT or Dcx-null primary E16 cortical neuron cultures 1 DIV (Stage 3) quantified in $\mathbf{C}$ and $\boldsymbol{C}^{\prime}$. Top image for each condition represents phalloidin staining. Bottom image for each condition represents nestin staining of the same neuron. Arrowheads indicate Nestin-positive GCs. Top, bottom, $\beta 3$ tubulin shown to both visualize the cells overall morphology and to confirm neuronal identity. $\boldsymbol{C}, \boldsymbol{C}^{\prime}, \boldsymbol{C}^{\prime \prime}$, Quantification of morphologic characteristics of nestin-negative and -positive neurons derived from WT or Dcx-null E16 cortex at 1 DIV (Stage 3). WT cells show nestin-dependent decrease in GC size, whereas $D C x$-null neurons do not. Axon length was not significantly different in any condition. When treated with $1 \mathrm{~nm}$ Sema3a for $5 \mathrm{~min}$, filopodial retraction in WT neurons was dependent on nestin expression. In Dcx-null neurons, there was a filopodia decrease after Sema3a treatment in nestin-positive and -negative neurons (independent of nestin). C, Statistical analysis was performed with one-way ANOVA with Sidak's multiple comparison correction (with pairs tested shown). $\boldsymbol{C}^{\prime}$, Statistical analysis was performed with one-way ANOVA with Tukey's multiple comparison correction. $\boldsymbol{C}^{\prime \prime}$, Statistical analysis was performed with one-way ANOVA with Sidak's multiple comparison correction (with pairs tested shown). C, $\boldsymbol{C}^{\prime}, \boldsymbol{C}^{\prime \prime}, n=4$ or 5 independent experiments with 20-30 cells counted in each set. $p$ values of $<0.05$ are indicated in bold. Error bars indicate SEM.

Figure 11. Model of nestin-mediated scaffolding of C $\mathrm{dk5}$ for modulation of DCX phosphorylation. Model for how nestin increases cdk5 phosphorylation of DCX by scaffolding: in the absence of nestin (-Nestin; top), non-phospho-DCX is bound to MTs and inefficiently phosphorylated by cdk5/p35. In the presence of nestin (+ Nestin scaffold; bottom), DCX becomes associated with $\mathrm{cdk} 5 / \mathrm{p} 35$, increasing the efficiency of DCX phosphorylation. DCX phosphorylation results in dissociation of DCX from MTs, thus allowing remodeling by additional factors (red bars). In this model, we also depict data from others that showed that the actin-associated SPN, which scaffolds PP1, potently dephosphorylates DCX. In our model, the level of phospho-DCX is determined by the relative opposing activities of the two regulatory complexes nestin/cdk5/p35 or SPN/PP1. However, in DCx-null neurons (bottom half), nestin no longer has an effect on sema3a sensitivity. Without DCX MT binding and stabilization, GCS retract and are sensitive to Sema3a independent of nestin. Nestin-mediated enhancement of Sema3a sensitivity is thus lost in Dcx-null neurons.

\section{DCX is a substrate for nestin-scaffolded cdk 5 in neurons}

Earlier work identified nestin as a p35 binding protein and showed that nestin modulated both the level and localization of cdk5 activity in C2C12 myotubes (Yang et al., 2011). In vivo, nestin depletion phenocopies $c d k 5$ deletion or roscovitine treatment in maintaining acetylcholine receptor clustering in agrin (AGD) mutants (Lin et al., 2005; Mohseni et al., 2011; Yang et al., 2011). However, the effect of nestin depletion on cdk5 activity 
(promoting or inhibiting) has varied between reports and assay systems and has been a point of contention (Sahlgren et al., 2006; Huang et al., 2009; Pallari et al., 2011; Yang et al., 2011; Lindqvist et al., 2017; J. Wang et al., 2018; Zhang et al., 2018). Since the relevant endogenous cdk5 substrate in myoblasts remains unknown, exogenous substrates (e.g., recombinant $\mathrm{H} 1$ histone protein) are commonly used for phosphorylation assays. The different interpretations are thus likely due to the use of exogenous substrates in the phosphorylation assays. Our work, for the first time, identifies a biologically relevant substrate for nestin-scaffolded cdk5, namely, DCX (Figs. 1, 3). Whether DCX is also the relevant substrate in myoblasts is not known, but it is intriguing that $D c x$-null mice also have developmental defects at the neuromuscular junction, as do human lissencephaly patients with $D c x$ mutations (Bourgeois et al., 2015). Our work suggests that nestin does not modulate cdk5/p35 kinase activity per se since nestin did not affect several other cdk5 substrates, nor was cdk5/p35 heterodimer formation affected (Figs. 1, 6D). Rather, we propose that in neurons nestin selectively increases cdk5-mediated DCX phosphorylation by providing a scaffold. We note that DCX may not be the only cdk5 substrate affected by nestin, especially in other tissues, but we focused our study on known neuronal cdk5 substrates to which specific antibodies to phosphoepitopes were available. Analysis of changes in the phosphoproteome of nestin null neurons will be required to more completely assess all the nestin-dependent phospho-proteins

\section{DCX as a downstream effector of nestin: a model for regulation of GC size}

How does nestin affect GC size via DCX? Three major processes are necessary for axon growth and guidance: protrusion, engorgement, and consolidation (Dent and Gertler, 2003). Protrusions toward positive guidance cues are mediated by actin-based filopodia and lamella, which serve as templates for subsequent MT infiltration and growth. These MTs stabilize the new actin-based structures and allow for transport of cell components through vesicular trafficking, which leads to the filling and engorgement of the advancing GC. The absence of DCX results in decreased stability and extent of protruding MTs (Jean et al., 2012). Last, the proximal part of the GC is consolidated, and MTs are bundled into new axon shaft. MT-binding proteins play essential roles in regulating the assembly dynamics and bundling extent of MTs in a spatially regulated manner and are critical regulators of axon growth. Phosphorylation and dephosphorylation are known to spatially regulate the activities of many MAPs, including DCX. We propose that nestin affects GC size by promoting the phosphorylation of DCX in the distal axon. Dephosphorylation of DCX has already been implicated in the consolidation step of axon growth (Bielas et al., 2007). Specifically, Bielas et al. (2007) showed that cdk5-mediated phosphorylation of DCX is reversed by the phosphatase PP1, which is scaffolded by the DCX-binding protein SPN (Tsukada et al., 2006). In extension of these earlier models, we propose that nestin scaffolds cdk5 and thereby promotes the phosphorylation of DCX, thus completing the regulatory cycle of DCX phosphorylation and dephosphorylation (Fig. 11). The regulation of the phosphorylation of DCX is thus driven by the balance of nestin/ $\mathrm{cdk} 5 / \mathrm{p} 35$ to increase phosphorylation and SPN/PP1 to decrease it; perturbation of either process results in abnormal MT organization (Schaar et al., 2004; Bielas et al., 2007; Dehmelt and Halpain, 2007). In our model, the level of phospho-DCX would be determined by the relative opposing activity of the two differently localized regulatory complexes (Fig. 11).
On a molecular level, we predict that the presence of nestin in GCs would result in more pS297-DCX, which has a decreased affinity for MTs. We were unable to stain neurons with the pS297DCX antibody reliably and could thus not test this idea directly. Since DCX binding to MTs stabilizes them (Moores et al., 2004), GCs with more pS297-DCX may have intrinsically less stable or more dynamic MTs. This notion is consistent with previous findings that GC size reflects MT dynamics. For instance, smaller GCs have intrinsically more dynamic MTs than larger GCs (Kiss et al., 2018). In addition, treatment with taxol, which stabilizes MTs, results in larger GCs (Gumy et al., 2013). Thus, we propose that a smaller GC represents a GC with more robust kinase/ phosphatase activity, which would result in more rapid cytoskeletal dynamics, morphologic turnover, and GC sculpting via changes in the phosphorylation of cytoskeletal associated proteins, including DCX. A smaller GC would thus correlate with a more robust response to a given relevant guidance cue as it is able to remodel the existing cytoskeleton more rapidly.

\section{A novel role for IFs in axon growth}

IFs have previously not been thought to be important for axon formation, much less axon guidance. Early studies had concluded that IFs were absent from the most distal and peripheral regions of the GC, and thus were ruled out early on as relevant players in regulating axon targeting (Dent and Gertler, 2003). However, the higher resolution and more sensitive imaging methods used here (Fig. 5B) reveal IFs in some peripheral regions of the GC, suggesting that this question should be revisited. Indeed, nestin mRNA has been consistently found to be enriched in neurites relative to the cell body (von Kügelgen and Chekulaeva, 2020). It remains thus an open question whether or not some IF proteins can affect axon guidance or outgrowth in some ways in vivo.

Nestin-containing IFs are indeed well localized to influence GC behavior. In support of our in vitro data, DCX and nestin coexpress in vivo while neurons are in the stage of axon positioning and orientation (Namba et al., 2014) (Fig. 5C,D), which are cdk5- and Sema3a-dependent processes (Polleux et al., 1998; Ohshima et al., 2007). Our own work shows striking accumulation of nestin in the distal axon shaft and even in some peripheral regions of the axonal GC (Fig. 5). Nestin colocalizes with a subset of DCX but is not identically distributed. This raises interesting future questions about why nestin is not present in dendrites, and whether this distribution is related to known differences in behavior of axonal and dendritic GCs (i.e., differential guidance cue sensitivity) (X. Wang et al., 2014).

In conclusion, this work highlights how the IF composition of neurons can alter intracellular signaling pathways and thus alter the cell's morphology. IFs have long been implicated as mediators and regulators of "cytoskeletal crosstalk" (Chang and Goldman, 2004; Michalczyk and Ziman, 2005), and our findings here represent an example of cytoskeletal crosstalk between IFs and MTs and their associated proteins. Our previous work has found that nestin expression correlated with small GC size and greater responsiveness to Sema3a. Based on our new work, we implicate modulation of DCX phosphorylation by cdk5 as a relevant molecular pathway downstream of neuronal nestin function. We thus posit that nestin sensitizes GCs to cdk 5 activity by scaffolding DCX. Based on our data, we propose the model that nestin affects GC morphology as a kinase regulator. In addition to this signaling role, nestin could also play a structural role as a cytoskeletal filament, but more work will be required to more fully elucidate nestin's role in neurons during development. 


\section{References}

Bahi-Buisson N, Souville I, Fourniol FJ, Toussaint A, Moores CA, Houdusse A, Lemaitre JY, Poirier K, Khalaf-Nazzal R, Hully M, Leger PL, Elie C, Boddaert N, Beldjord C, Chelly J, Francis F, SBH-LIS European Consortium (2013) New insights into genotype-phenotype correlations for the doublecortin-related lissencephaly spectrum. Brain 136:223-244.

Benson DL, Mandell JW, Shaw G, Banker G (1996) Compartmentation of alpha-internexin and neurofilament triplet proteins in cultured hippocampal neurons. J Neurocytol 25:181-196.

Bielas SL, Serneo FF, Chechlacz M, Deerinck TJ, Perkins GA, Allen PB, Ellisman MH, Gleeson JG (2007) Spinophilin facilitates dephosphorylation of doublecortin by PP1 to mediate microtubule bundling at the axonal wrist. Cell 129:579-591.

Bott CJ, Winckler B (2020) Intermediate filaments in developing neurons: beyond structure. Cytoskeleton (Hoboken) 77:110-128.

Bott CJ, Johnson CG, Yap CC, Dwyer ND, Litwa KA, Winckler B (2019) Nestin in immature embryonic neurons affects axon growth cone morphology and Semaphorin3a sensitivity. Mol Biol Cell 30:1214-1229.

Bourgeois F, Messéant J, Kordeli E, Petit JM, Delers P, Bahi-Buisson N, Bernard V, Sigoillot SM, Gitiaux C, Stouffer M, Francis F, Legay C (2015) A critical and previously unsuspected role for doublecortin at the neuromuscular junction in mouse and human. Neuromusc Disord 25:461-473.

Brown A (2013) Axonal transport. In: Neuroscience in the 21st century, (Donald W. Pfaff, ed) pp 255-308. New York: Springer.

Chang L, Goldman RD (2004) Intermediate filaments mediate cytoskeletal crosstalk. Nat Rev Mol Cell Biol 5:601-613.

Cheung ZH, Ip NY (2012) Cdk5: a multifaceted kinase in neurodegenerative diseases. Trends Cell Biol 22:169-175.

Contreras-Vallejos E, Utreras E, Bórquez DA, Prochazkova M, Terse A, Jaffe H, Toledo A, Arruti C, Pant HC, Kulkarni AB, González-Billault C (2014) Searching for novel Cdk5 substrates in brain by comparative phosphoproteomics of wild type and Cdk5-/- mice. PLoS One 9:e90363-13.

Dehmelt L, Halpain S (2007) Neurite outgrowth: a flick of the wrist. Curr Biol 17:R611.

Dent EW, Gertler FB (2003) Cytoskeletal dynamics and transport in growth cone motility and guidance. Neuron 40:209-227.

Dent EW, Gupton SL, Gertler FB (2011) The growth cone cytoskeleton in axon outgrowth and guidance. Cold Spring Harbor Perspect Biol 3: a001800.

Dhavan R, Tsai L (2001) A decade of Cdk5. Nat Rev Mol Cell Biol 2:749-759.

Friocourt G, Koulakoff A, Chafey P, Boucher D, Fauchereau F, Chelly J, Francis F (2003) Doublecortin functions at the extremities of growing neuronal processes. Cereb Cortex 13:620-626.

Ge W, He F, Kim KJ, Blanchi B, Coskun V, Nguyen L, Wu X, Zhao J, Heng JI, Martinowich K, Tao J, Wu H, Castro D, Sobeih MM, Corfas G, Gleeson JG, Greenberg ME, Guillemot F, Sun YE (2006). Coupling of cell migration with neurogenesis by proneural bHLH factors. Proc Natl Acad Sci USA 103:1319-1324.

Geraldo S, Gordon-Weeks PR (2009) Cytoskeletal dynamics in growth-cone steering. J Cell Sci 122:3595-3604.

Gleeson JG, Lin PT, Flanagan LA, Walsh CA (1999) Doublecortin is a microtubule-associated protein and is expressed widely by migrating neurons. Neuron 23:257-271.

Goldsmith EJ, Akella R, Min X, Zhou T, Humphreys JM (2007) Substrate and docking interactions in Ser/Thr protein kinases. Chem Rev 107:5065-5081.

Gumy LF, Chew DJ, Tortosa E, Katrukha EA, Kapitein LC, Tolkovsky AM, Hoogenraad CC, Fawcett JW (2013) The Kinesin-2 family member $\mathrm{KIF} 3 \mathrm{C}$ regulates microtubule dynamics and is required for axon growth and regeneration. J Neurosci 33:11329-11345.

Hirasawa M, Ohshima T, Takahashi S, Longenecker G, Honjo Y, Veeranna Pant HC, Mikoshiba K, Brady R, Kulkarni AB (2004) Perinatal abrogation of Cdk5 expression in brain results in neuronal migration defects. Proc Natl Acad Sci USA 101:6249-6254.

Huang YL, Wu CM, Shi GY, Wu GC, Lee H, Jiang MJ, Wu HL, Yang HY (2009) Nestin serves as a prosurvival determinant that is linked to the cytoprotective effect of epidermal growth factor in rat vascular smooth muscle cells. J Biochem 146:307-315.

Jean DC, Baas PW, Black MM (2012) A novel role for doublecortin and doublecortin-like kinase in regulating growth cone microtubules. Hum Mol Genet 21:5511-5527.
Kapitein LC, Hoogenraad CC (2015) Building the neuronal microtubule cytoskeleton. Neuron 87:492-506

Kawauchi T (2014) Cdk5 regulates multiple cellular events in neural development, function and disease. Dev Growth Differ 56:335-348.

Kiss A, Fischer I, Kleele T, Misgeld T, Propst F (2018) Neuronal growth cone size-dependent and -independent parameters of microtubule polymerization. Front Cell Neurosci 12:1-15.

Laser-Azogui A, Kornreich M, Malka-Gibor E, Beck R (2015) Neurofilament assembly and function during neuronal development. Curr Opin Cell Biol 32:92-101.

Lin W, Dominguez B, Yang J, Aryal P, Brandon EP, Gage FH, Lee K (2005) Neurotransmitter acetylcholine negatively regulates neuromuscular synapse formation by a Cdk5-dependent mechanism. Neuron 46:569-579.

Lin W, Szaro B (1995) Neurofilaments help maintain normal morphologies and support elongation of neurites in Xenopus laevis cultured embryonic spinal cord neurons. J Neurosci 15:8331-8344.

Lin W, Szaro BG (1996) Effects of intermediate filament disruption on the early development of the peripheral nervous system of Xenopus laevis. Dev Biol 179:197-211.

Lindqvist J, Torvaldson E, Gullmets J, Karvonen H, Nagy A, Taimen P, Eriksson JE (2017) Nestin contributes to skeletal muscle homeostasis and regeneration. J Cell Sci 130:2833-2842.

Manning BD, Cantley LC (2002) Hitting the target: emerging technologies in the search for kinase substrates. Sci STKE 162:1-5

Menon S, Gupton SL (2016) Building blocks of functioning brain: cytoskeletal dynamics in neuronal development. Int Rev Cell Mol Biol 3:183245.

Michalczyk K, Ziman M (2005) Nestin structure and predicted function in cellular cytoskeletal organisation. Histol Histopathol 20:665-671.

Miller KE, Suter DM (2018) An integrated cytoskeletal model of neurite outgrowth. Front Cell Neurosci 12:1-19.

Mohseni P, Sung HK, Murphy AJ, Laliberte CL, Pallari HM, Henkelman M, Georgiou J, Xie G, Quaggin SE, Thorner PS, Eriksson JE, Nagy A (2011) Nestin is not essential for development of the CNS but required for dispersion of acetylcholine receptor clusters at the area of neuromuscular junctions. J Neurosci 31:11547-11552.

Moores CA, Perderiset M, Francis F, Chelly J, Houdusse A, Milligan RA (2004) Mechanism of microtubule stabilization by doublecortin. Mol Cell 14:833-839.

Moslehi M, Ng DC, Bogoyevitch MA (2017) Dynamic microtubule association of Doublecortin X (DCX) is regulated by its C-terminus. Sci Rep 7:71-11.

Namba T, Kibe Y, Funahashi Y, Nakamuta S, Takano T, Ueno T, Shimada A, Kozawa S, Okamoto M, Shimoda Y, Oda K, Wada Y, Masuda T, Sakakibara A, Igarashi M, Miyata T, Faivre-Sarrailh C, Takeuchi K, Kaibuchi K (2014) Pioneering axons regulate neuronal polarization in the developing cerebral cortex. Neuron 81:814-829.

Ng T, Ryu JR, Sohn JH, Tan T, Song H, Ming GL, Goh EL (2013) Class 3 Semaphorin mediates dendrite growth in adult newborn neurons through Cdk5/FAK pathway. PLoS One 8:e65572.

Nishimura YV, Shikanai M, Hoshino M, Ohshima T, Nabeshima Y, Mizutani K, Nagata K, Nakajima K, Kawauchi T (2014) Cdk5 and its substrates, Dcx and p27 kip1, regulate cytoplasmic dilation formation and nuclear elongation in migrating neurons. Development 141:3540-3550.

Nixon RA, Shea TB (1992) Dynamics of neuronal intermediate filaments: a developmental perspective. Cell Motil Cytoskeleton 22:81-91.

Ohshima T, Ward JM, Huht C, Longenecker G, Veeranna ant HC, Brady R, Martin LJ, Kulkarni AB (1996) Targeted disruption of the cyclin-dependent kinase 5 gene results in abnormal corticogenesis, neuronal pathology and perinatal death. Proc Natl Acad Sci USA 93:11173-11178.

Ohshima T, Hirasawa M, Tabata H, Mutoh T, Adachi T, Suzuki H, Saruta K, Iwasato T, Itohara S, Hashimoto M, Nakajima K, Ogawa M, Kulkarni AB, Mikoshiba K (2007) Cdk5 is required for multi-to-bipolar transition during radial neuronal migration and proper dendrite development of pyramidal neurons in the cerebral cortex. Development 134:2273-2282.

Pallari HM, Lindqvist J, Torvaldson E, Ferraris SE, He T, Sahlgren C, Eriksson JE (2011) Nestin as a regulator of Cdk5 in differentiating myoblasts. Mol Biol Cell 22:1539-1549.

Park D, Xiang AP, Mao FF, Zhang L, Di CG, Liu XM, Shao Y, Ma BF, Lee JH, Ha KS, Walton N, Lahn BT (2010) Nestin is required for the proper self-renewal of neural stem cells. Stem Cells 28:2162-2171. 
Pennypacker K, Fischer I, Levitt P (1991) Early in vitro genesis and differentiation of axons and dendrites by hippocampal neurons analyzed quantitatively with neurofilament- $\mathrm{H}$ and microtubule-associated protein 2 antibodies. Exp Neurol 111:25-35.

Petrovic M, Schmucker D (2015) Axonal wiring in neural development: target-independent mechanisms help to establish precision and complexity. Bioessays 37:996-1004.

Polleux F, Giger R, Ginty D, Kolodkin A, Chosh A (1998) Patterning of cortical efferent projections by semaphorin-neuropolin interactions. Science 282:1904-1906.

Sahlgren CM, Mikhailov A, Vaittinen S, Pallari H, Kalimo H, Pant HC, Eriksson JE (2003) Cdk5 regulates the organization of Nestin and its association with p35. Mol Cell Biol 23:5090-5106.

Sahlgren CM, Pallari HM, He T, Chou YH, Goldman RD, Eriksson JE (2006) A Nestin scaffold links Cdk5/p35 signaling to oxidant-induced cell death. EMBO J 25:4808-4819.

Sakakibara A, Hatanaka Y (2015) Neuronal polarization in the developing cerebral cortex. Front Neurosci 9:1-10.

Sapir T, Horesh D, Caspi M, Atlas R, Burgess HA, Wolf SG, Francis F, Chelly J, Elbaum M, Pietrokovski S, Reiner O (2000) Doublecortin mutations cluster in evolutionarily conserved functional domains. Hum Mol Genet 9:703-712.

Schaar BT, Kinoshita K, McConnell SK (2004) Doublecortin microtubule affinity is regulated by a balance of kinase and phosphatase activity at the leading edge of migrating neurons. Neuron 41:203-213.

Shaw G, Banker G, Weber K (1985) An immunofluorescence study of neurofilament protein expression by developing hippocampal neurons in tissue culture. Eur J Cell Biol 39:205-216.

Shin E, Kashiwagi Y, Kuriu T, Iwasaki H, Tanaka T, Koizumi H, Gleeson JG, Okabe S (2013) Doublecortin-like kinase enhances dendritic remodelling and negatively regulates synapse maturation. Nat Commun 4:1440.

Shmueli A, Gdalyahu A, Sapoznik S, Sapir T, Tsukada M, Reiner O (2006) Site-specific dephosphorylation of doublecortin (DCX) by protein phosphatase 1 (PP1). Mol Cell Neurosci 32:15-26.

Su PH, Chen CC, Chang YF, Wong ZR, Chang KW, Huang BM, Yang HY (2013) Identification and cytoprotective function of a novel Nestin isoform, Nes-S, in dorsal root ganglia neurons. J Biol Chem 288:8391-8404.

Szaro BE, Grant P, Lee VM, Gainer H (1991) Inhibition of axonal development after injection of neurofilament antibodies into a Xenopus zaeuis embryo. J Comp Neurol 308:576-585.

Tamariz E, Varela-Echavarría A (2015) The discovery of the growth cone and its influence on the study of axon guidance. Front Neuroanat 9:1-9.

Tanaka T, Serneo FF, Higgins C, Gambello MJ, Wynshaw-Boris A, Gleeson JG (2004) Lis1 and doublecortin function with dynein to mediate coupling of the nucleus to the centrosome in neuronal migration. J Cell Biol 165:709-721.

Tanaka T, Serneo FF, Tseng HC, Kulkarni AB, Tsai LH, Gleeson JG (2004) Cdk5 phosphorylation of doublecortin Ser297 regulates its effect on neuronal migration. Neuron 41:215-227.

Tsukada M, Prokscha A, Ungewickell E, Eichele G (2005) Doublecortin association with actin filaments is regulated by neurabin II. J Biol Chem 280:11361-11368.

Tsukada M, Prokscha A, Eichele G (2006) Neurabin II mediates doublecortin-dephosphorylation on actin filaments. Biochem Biophys Res Commun 343:839-847.
Uchida A, Brown A (2004) Arrival, reversal, and departure of neurofilaments at the tips of growing axons. Mol Biol Cell 15:4215-4225.

Vitriol EA, Zheng JQ (2012) Growth cone travel in space and time: the cellular ensemble of cytoskeleton, adhesion, and membrane. Neuron 73:10681080 .

von Kügelgen N, Chekulaeva M (2020) Conservation of a core neurite transcriptome across neuronal types and species. Wiley Interdiscip Rev RNA 2020:e1590.

Walker KL, Yoo HK, Undamatla J, Szaro BG (2001) Loss of neurofilaments alters axonal growth dynamics. J Neurosci 21:9655-9666.

Wang J, Huang Y, Cai J, Ke Q, Xiao J, Huang W, Li H, Qiu Y, Wang Y, Zhang B, Wu H, Zhang Y, Sui X, Bardeesi AS, Xiang AP (2018) A Nestin-cyclin-dependent kinase 5-dynamin-related protein 1 axis regulates neural stem/progenitor cell stemness via a metabolic shift. Stem Cells 36:589-601

Wang L, Ho C, Sun D, Liem RK, Brown A (2000) Rapid movement of axonal neurofilaments interrupted by prolonged pauses. Nat Cell Biol 2:137141.

Wang X, Sterne GR, Ye B (2014) Regulatory mechanisms underlying the differential growth of dendrites and axons. Neurosci Bull 30:557-568.

Wilhelmsson U, Lebkuechner I, Leke R, Marasek P, Yang X, Antfolk D, Chen M, Mohseni P, Lasic E, Bobnar ST, Stenovec M, Zorec R, Nagy A, Sahlgren C, Pekna M, Pekny M (2019) Nestin regulates neurogenesis in mice through notch signaling from astrocytes to neural stem cells. Cereb Cortex 29:4050-4017.

Xie Z, Samuels BA, Tsai LH (2006) Cyclin-dependent kinase 5 permits efficient cytoskeletal remodeling: a hypothesis on neuronal migration. Cereb Cortex 16:64-68.

Yabe JT, Chan WK, Wang FS, Pimenta A, Ortiz DD, Shea TB (2003) Regulation of the transition from vimentin to neurofilaments during neuronal differentiation. Cell Motil Cytoskeleton 56:193-205.

Yamada KM, Spooner BS, Wessells NK (1970) Axon growth: roles of microfilaments and microtubules. Proc Natl Acad Sci USA 66:1206-1212.

Yamamoto N, Tamada A, Murakami F (2002) Wiring of the brain by a range of guidance cues. Prog Neurobiol 68:393-407.

Yang J, Dominguez B, de Winter F, Gould TW, Eriksson JE, Lee KF (2011) Nestin negatively regulates postsynaptic differentiation of the neuromuscular synapse. Nat Neurosci 14:324-330.

Yap CC, Digilio L, Kruczek K, Roszkowska M, Fu X, Liu JS, Winckler XB (2018) A dominant dendrite phenotype caused by the disease-associated G253D mutation in doublecortin (DCX) is not due to its endocytosis defect. J Biol Chem 293:18890-18902.

Yap CC, Digilio L, McMahon L, Roszkowska M, Bott CJ, Kruczek K, Winckler XB (2016) Different doublecortin (DCX) patient alleles show distinct phenotypes in cultured neurons. J Biol Chem 291:26613-26626.

Yap CC, Vakulenko M, Kruczek K, Motamedi B, Digilio L, Liu JS, Winckler B (2012) Doublecortin (DCX) mediates endocytosis of neurofascin independently of microtubule binding. J Neurosci 32:7439-7453.

Yuan A, Rao MV (2012) Neurofilaments at a glance. J Cell Sci 125:32573263.

Zhang Y, Wang J, Huang W, Cai J, Ba J, Wang Y, Ke Q, Huang Y, Liu X, Qiu Y, Lu Q, Sui X, Shi Y, Wang T, Shen H, Guan Y, Zhou Y, Chen Y, Wang M, Xiang AP (2018) Nuclear Nestin deficiency drives tumor senescence via lamin A/C-dependent nuclear deformation. Nat Commun 9:1-14. 